\title{
Modelagem de riscos proporcionais para dados de sobrevivência com estrutura hierárquica
}

\author{
Gisela Tunes da Silva
}

\author{
Dissertação apresentada ao \\ Instituto de Matemática e Estatística \\ da Universidade de São Paulo \\ como requisito parcial \\ para a obtenção do grau de \\ Mestre em Estatística
}

Área de Concentração: Estatística

Orientador: Prof. Dr. Antonio Carlos Pedroso de Lima

Durante o curso de Mestrado e elaboração desse trabalho o autor recebeu apoio financeiro da CAPES

- São Paulo, 25 de julho de 2001 - 
Aos meus Pais. 


\section{Agradecimentos}

Ao Antonio Carlos, pela orientação, apoio e incentivo.

À Édina Miazaki, que muito me ajudou na graduação e sempre me incentivou a prosseguir os estudos.

Ao Josué, pelos momentos felizes e por compreender todos os bolos que dei.

À Larissa e ao Xerox, por terem paciência de me ouvir, mesmo sem entenderem nada.

Ao Tiago, por brincar de carrinho comigo, e à Gabriela, pelo Tiago.

Aos amigos que estiveram comigo, especialmente Fábio, Mineirinho, Arturo, Ithana, Joanlise, Lia e todos os outros que não saíram na minha amostra. 


\section{Resumo}

As técnicas mais difundidas em análise de sobrevivência supõem independência entre as observações. No entanto, em muitos problemas práticos esta suposição não é adequada, pela própria natureza dos dados. Uma maneira de abordar o problema é a utilização de modelos multiníveis, que são desenvolvidos para dados com estrutura hierárquica. Nestes modelos, a inclusão de coeficientes aleatórios faz com que o modelo possa incorporar dependências entre observações. Neste trabalho, foram estudados modelos de sobrevivência multiníveis em que os coeficientes apresentam distribuição normal, considerando-se uma abordagem paramétrica (modelos exponencial e Weibull) e uma abordagem baseada no modelo de regressão de Cox. A estimação estudada baseia-se na metodologia desenvolvida para modelos lineares generalizados. São apresentados dois métodos de estimação, que foram utilizados para analisar três conjuntos de dados. Além disso, é utilizado o método bootstrap para verificação de hipóteses de interesse sobre os parâmetros.

\section{Abstract}

In Survival Analysis, the methodology usually considered assume independence among the observations. Nevertheless, it is not uncommon that such assumption is not adequate. In order to incorporate the dependence in the model, one may use multilevel models. These models were developed to analyze data with hierarchical structure. The main difference between multilevel models and the usual approach is the use of random coefficients in the former. In this work, we consider multilevel models in Survival Analysis in which the random coefficients are normally distributed. We focus on parametric models, especifically the exponential and the Weibull distributions. We also consider the semiparametric approach based on the Cox proportional hazards model. The estimation of parameters is based on mixed generalized linear models. We present two different estimation methods, which are used to analyze three data sets. In addition, the bootstrap is used in order to get some insight on hypothesis testing. 


\section{Sumário}

1 Introdução $\quad 1$

2 Modelos Multiníveis 4

2.1 Introdução . . . . . . . . . . . . . . . . . . 4

2.2 Histórico dos modelos multiníveis . . . . . . . . . . . . . . . 6

2.3 Modelos Lineares Multiníveis . . . . . . . . . . . . . . . 8

2.4 Modelos Lineares Multiníveis Generalizados . . . . . . . . . . . . . . 11

3 Modelos Multiníveis de riscos proporcionais $\quad 16$

3.1 Introdução . . . . . . . . . . . . . . . . . 16

3.2 Modelos de riscos proporcionais . . . . . . . . . . . . . . 17

3.2.1 Modelos paramétricos de riscos proporcionais . . . . . . . . . 19

3.2 .2 Modelo semiparamétrico de Cox . . . . . . . . . . . . . . 24

3.3 Inferência nos modelos Multiníveis . . . . . . . . . . . . . . . . 29

3.3.1 Quase Verossimilhança Penalizada . . . . . . . . . . . . . 31 
3.3.2 Quase Verossimilhança Marginal . . . . . . . . . . . . 38

3.3.3 Correção de viés da quase verossimilhança penalizada . . . . . . . 42

3.3.4 Testes de hipóteses - aplicação do método Bootstrap . . . . . . . . . 45

4 Aplicações $\quad 49$

4.1 Introdução . . . . . . . . . . . . . . . . . . . 49

4.2 Comparação de selantes . . . . . . . . . . . . . . . 50 50

4.3 Doença granulomatosa crônica da infância . . . . . . . . . . . . . 55

4.4 Avaliação de uma droga carcinogênica em ratos . . . . . . . . . . . . . 60

5 Conclusão $\quad 64$

$\begin{array}{lll}\text { A Cálculo da expressão aproximada da PQL } & 67\end{array}$

$\begin{array}{ll}\text { Referências Bibliográficas } & 70\end{array}$ 


\section{Lista de Tabelas}

4.1 Resultados para o exemplo de selantes . . . . . . . . . . . . . . . 52

4.2 Resultados do ajuste exponencial para o exemplo de selantes . . . . . . . . 54

4.3 Resultados do ajuste do modelo Weibull para o exemplo de selantes . . . . 55

4.4 Resultados para o exemplo da doença granulomatosa . . . . . . . . . 57

4.5 Resultados do ajuste exponencial para o exemplo da doença granulomatosa 59

4.6 Resultados do ajuste do modelo Weibull para o exemplo da doença granulomatosa . . . . . . . . . . . . . . . . . . . 59

4.7 Resultados para o exemplo de tumores em ratos . . . . . . . . . . . . . 61

4.8 Resultados do ajuste exponencial para o exemplo de tumores em ratos . . . 63

4.9 Resultados do ajuste do modelo Weibull para o exemplo de tumores em ratos 63 


\section{Capítulo 1}

\section{Introdução}

As técnicas mais difundidas na análise de dados de sobrevivência supõem independência entre as observações. Na prática, entretanto, é muito comum encontrar dados que não satisfazem a essa suposição. A utilização de um modelo cujas suposições não são satisfeitas pode levar a conclusões incorretas. Existem vários exemplos, não só na área de análise de sobrevivência, em que se verifica que são incorretas as conclusões obtidas com base em análises cujas suposições não são válidas. Assim sendo, é necessário generalizar os modelos que supõem independência para que sejam incorporadas possíveis dependências entre observações.

Os modelos multiníveis foram desenvolvidos para dados que apresentam um certo tipo de estrutura, especificamente, uma estrutura hierárquica. Conforme será exposto com mais detalhes no capítulo 2, em dados com essa estrutura, as observações podem ser naturalmente agrupadas, gerando estruturas de dependência particulares. Os modelos multiníveis surgiram para analisar dados dessa natureza em modelos lineares. Como existem muitos problemas em análise de sobrevivência com estrutura hierárquica, tornouse interessante aplicar a metodologia desenvolvida para os modelos multiníveis em dados 
dessa natureza.

Um exemplo que será bastante discutido neste trabalho é o estudo feito na área de odontologia, publicado por Grande, Lima, Filho \& Witzel (2000), em que o objetivo principal é comparar dois tipos diferentes de selantes utilizados na prevenção de cáries. Um dos selantes (Delton) é o normalmente empregado, utilizado nos consultórios dentários, e o outro (OptiBond) é um material adesivo, normalmente utilizado com outra finalidade. Nesse estudo, os selantes foram aplicados em vários dentes de 37 crianças, com idade entre 11 e 17 anos. Na metade dos dentes de cada criança aplicou-se, via aleatorização, o selante Delton e na outra metade, o Optibond. No total, foram aplicados selantes em 171 dentes (sem cáries), dos quais 86 receberam o Delton e 85 receberam o ObtiBond. Durante o estudo, foram feitas avaliações periódicas para verificar se havia ou não queda dos selantes aplicados. Quando havia queda do selante, o dente não era mais observado, mas os demais dentes daquela criança continuavam a ser observados. Ao término do estudo, os selantes de muitos dentes ainda não haviam caído, caracterizando, dessa forma, observações censuradas. A variável resposta do estudo era o tempo (em meses) até a queda parcial ou total do selante. Outras variáveis também foram observadas: tipo de dente (molar ou pré-molar), idade e sexo. Como em uma criança foram observados vários dentes, pode-se agrupar as observações por crianças, constituindo-se a estrutura hierárquica. No artigo de Grande et al. (2000), a análise foi feita supondo-se independência entre as observações e os resultados mostraram que o OptiBond teve um desempenho melhor. Os fatores idade e sexo não apresentaram diferenças significativas e os dentes molares apresentaram menor retenção de selantes. Esse conjunto de dados será analisado novamente nesse trabalho, mas agora sem a suposição de independência entre as observações. No capítulo 4 são apresentados outros exemplos de dados com estrutura hierárquica.

Existem na literatura alguns modelos para analisar dados de sobrevivência com dependência, como o descrito acima. Um exemplo é a classe de modelos de fragilidade, 
apresentada de forma detalhada em Hougaard (2000). Os modelos de fragilidade são modelos de efeitos aleatórios, assim como os modelos multiníveis, em que se tem uma fonte de variação individual e uma fonte de variação de grupo, representada por uma variável aleatória, conhecida como variável de fragilidade. Esses modelos, entretanto, ainda estão sendo estudados e são alvo de muita discussão.

Neste trabalho, serão utilizados os modelos multiníveis para resolver o problema da dependência entre observações. Esses modelos também são modelos de efeitos aleatórios, mas a abordagem é um pouco diferente do que no caso dos modelos de fragilidade. Em particular, os modelos de sobrevivência considerados são os modelos paramétricos (exponencial e Weibull) e o modelo de riscos proporcionais de Cox. No capítulo 2, é introduzida a modelagem multinível de forma geral. O capítulo 3 trata dos modelos multiníveis em análise de sobrevivência, incluindo estimação dos parâmetros e testes de hipóteses. Por fim, no capítulo 4, são apresentados exemplos de dados com estrutura hierárquica que foram analisados por meio de modelos multiníveis. 


\section{Capítulo 2}

\section{Modelos Multiníveis}

\subsection{Introdução}

Os modelos multiníveis, também conhecidos como modelos hierárquicos, foram desenvolvidos para analisar dados com estrutura hierárquica, isto é, dados cujas unidades de observação podem ser agrupadas segundo algum critério. No exemplo dos selantes, apresentado na introdução, os dentes podem ser agrupados por crianças, constituindo assim a estrutura hierárquica.

$\mathrm{Na}$ terminologia de modelos multiníveis, têm-se unidades agrupadas em diferentes níveis. Os dentes representam as unidades do nível 1, que estão agrupados em crianças, que, por sua vez, constituem as unidades do nível 2. Se, no exemplo dos dentes, as crianças estivessem agrupadas por família (no caso de haver irmãos no estudo, por exemplo) ou classe social, os dados teriam uma estrutura de três níveis, com as famílias ou classes sociais constituindo as unidades do nível 3.

A diferença fundamental entre os modelos multiníveis e os modelos de regressão 
usuais é a existência de coeficientes aleatórios no modelo. A inclusão dos coeficientes aleatórios faz com que o modelo incorpore a dependência entre as observações. Na verdade, supõe-se, por exemplo, que as crianças constituem uma amostra aleatória de uma população, cuja característica de interesse apresenta alguma distribuição de probabilidade (em geral, considera-se a distribuição normal).

Os modelos multiníveis são mais adequados para a análise de dados com estrutura hierárquica do que os modelos de regressão usuais porque procuram incorporar uma possível dependência entre observações pertencentes a um mesmo grupo. Por exemplo, selantes aplicados aos dentes de uma mesma criança podem apresentar desempenhos (em termos de resistência) mais próximos, pelo fato de os selantes estarem submetidos às mesmas condições (por exemplo, PH da boca, força na mastigação, uso ou não de goma de mascar, fatores genéticos etc.). Assim, a correlação entre desempenhos de selantes de uma mesma criança deve ser levada em consideração na modelagem.

Antes do surgimento dos modelos multiníveis, uma forma muito utilizada para analisar dados com estrutura hierárquica era por meio de agregação dos dados (para maiores detalhes, ver Hox (1995) e Draper (1995) ). Nesta abordagem, diferentes dados de unidades menores são combinados em unidades maiores, por intermédio de medidas resumo, como a média, o que pode acarretar uma indesejável perda de informação. Uma outra maneira também utilizada era simplesmente desconsiderar a estrutura hierárquica. As unidades do nível são consideradas observações independentes e, portanto, as correlações não são incorporadas no modelo.

É interessante observar que dados com medidas repetidas e dados longitudinais também podem ser considerados hierárquicos. Nesse caso, cada observação feita em um mesmo indivíduo ao longo do tempo constituiria a unidade do nível 1 e os indivíduos, a unidade do nível 2. 
Apesar de ser abordado aqui apenas o caso de dados com estrutura hierárquica, deve-se ressaltar que os modelos multiníveis também podem ser utilizados para dados com classificação cruzada (crossed classifications), conforme discutido em Goldstein (1995). No exemplo dos selantes, se o outro selante fosse aplicado no mesmo dente após a queda do primeiro, os dados teriam uma estrutura cruzada.

\subsection{Histórico dos modelos multiníveis}

Diversos casos estudados na área de ciências sociais apresentam estrutura hierárquica. Os exemplos mais citados na literatura são na área educacional, em estudos para avaliar a eficácia de técnicas de ensino. Em geral, tem-se uma amostra aleatória de escolas, de professores em cada escola e finalmente de alunos, caracterizando a estrutura hierárquica. Motivada principalmente por esse tipo de dados, a formulação geral dos modelos multiníveis lineares surgiu na década de 70 (Lindley \& Smith 1972) e ganhou grande impulso com o desenvolvimento do algoritmo EM (Dempster, Laird \& Rubin 1977).

O uso de modelos lineares mistos permite a incorporação da estrutura de dependência das observações de forma mais adequada. Várias formas de estimação para essa classe de modelos têm sido desenvolvidas. Aitkin, Anderson \& Hinde (1981) reanalisam os dados apresentados em Bennet (1976) considerando a estrutura hierárquica e utilizam o método de máxima verossimilhança e máxima verossimilhança restrita para a estimação dos parâmetros. Esses modos de estimação são também discutidos em Harville (1977), porém para o caso linear. Goldstein (1986) propõe a estimação utilizando o método de mínimos quadrados generalizados iterativo (IGLS) e mostra que esse procedimento é equivalente ao método de máxima verossimilhança no caso normal. Ainda, em Longford (1987), um algoritmo utilizando o método Scoring de Fisher para obter estimativas de máxima verossimilhança é desenvolvido. Mais recentemente, Walker (1996) trabalha com 
modelos não lineares com efeitos aleatórios, supondo distribuição normal para a variável resposta. O autor desenvolve o algoritmo EM para obter estimativas de máxima verossimilhança, utilizando métodos de Monte Carlo no passo E do algoritmo.

A extensão dos modelos com efeitos aleatórios para outras distribuições além da normal não é imediata. A aplicação do método de máxima verossimilhança é difícil, pois chega-se a integrais intratáveis analiticamente para problemas um pouco mais complicados. Em alguns artigos, são desenvolvidos métodos de estimação para casos específicos, como é o caso de Gilmour, Anderson \& Rae (1985), que desenvolve um método de estimação para o caso da distribuição binomial.

Uma abordagem mais geral é apresentada por Goldstein (1991), que considera distribuições pertencentes à família exponencial e propõe um método de estimação para o caso em que os coeficientes aleatórios possuem distribuição normal. Escrevendo a variável resposta como sendo igual a uma função $f$ (não linear) do preditor linear (que contém efeitos fixos e aleatórios), o método proposto consiste, basicamente, em linearizar a função $f$ através da expansão de Taylor e aplicar algum dos métodos desenvolvidos para o caso linear. Um algoritmo simples, proposto por Schall (1991), produz estimativas de máxima verossimilhança aproximadas quando a distribuição dos coeficientes aleatórios pertencem à família exponencial.

Breslow \& Clayton (1993) propõem uma outra forma de estimação também para o caso em que os coeficientes aleatórios possuem distribuição normal, utilizando a aproximação de Laplace para integrais envolvidas . Eles obtêm equações baseadas na quaseverossimilhança penalizada para os coeficientes fixos e pseudo-verossimilhança para os parâmetros da variância. Por meio de simulações e exemplos, verificaram que esse método, denominado pelos autores de PQL, produz bons resultados, mas tende a subestimar os parâmetros da variância e dos efeitos fixos em alguns casos de dados com distribuição binomial. Expressões gerais para o viés assintótico de alguns estimadores aproximados, 
incluindo PQL, foram obtidas em Breslow \& Lin (1995) e Lin \& Breslow (1996) e fatores de correção foram propostos.

Uma abordagem Bayesiana é apresentada por Zeger \& Karim (1991), que consideram o uso do amostrador de Gibbs para estimar parâmetros em modelos lineares generalizados no caso em que os coeficientes aleatórios possuem distribuição normal.

No caso específico de análise de sobrevivência, uma forma que vem sendo estudada para incorporar a dependência entre as observações são os modelos de fragilidade introduzidos por Clayton (1978). Os modelos de fragilidade utilizam uma ou mais variáveis aleatórias não observáveis (variável de fragilidade), introduzida na função de risco de forma multiplicativa ou aditiva. Os modelos mais estudados são os modelos de fragilidade em que a variável de fragilidade possui distribuição gama. Para um apanhado desse caso, ver Chicarino (1999) e Hougaard (2000) .

A utilização de modelos multiníveis em análise de sobrevivência é um pouco mais recente. Para a estimação dos parâmetros, Goldstein (1995) utiliza um resultado que mostra a equivalência da verossimilhança de modelos de sobrevivência (alguns paramétricos e o semiparamétrico) e a verossimilhança de Poisson, conforme discutido em Aitkin, Anderson, Francis \& Hinde (1989). Assim sendo, os resultados obtidos para modelos lineares generalizados mistos podem ser utilizados para a estimação de modelos multiníveis em análise de sobrevivência. Esses resultados serão discutidos com detalhe no capítulo 3.

\subsection{Modelos Lineares Multiníveis}

Nesta seção, será apresentada a formulação geral dos modelos multiníveis lineares, para formalizar as idéias já expostas e introduzir a notação que será utilizada.

Considere um conjunto de dados com 2 níveis apenas. Seja $y_{i j}$ a variável resposta 
da $i$-ésima unidade do nível 1 e $j$-ésima unidade do nível 2 e suponha que exista apenas uma covariável de interesse. O modelo pode, então, ser escrito como

$$
y_{i j}=\beta_{0 j}+\beta_{1 j} x_{i j}+e_{i j}
$$

$\operatorname{com} E\left(e_{i j}\right)=0$ e $\operatorname{var}\left(e_{i j}\right)=\sigma^{2}$. Se $\beta_{0 j}=\beta_{0}$ e $\beta_{1 j}=\beta_{1}, j=1, \ldots, J$, tem-se o modelo de regressão usual.

Suponha, agora, que no modelo (2.1) toma-se $\beta_{1 j}=\beta_{1}$ e $\beta_{0 j}=\beta_{0}+b_{0 j}$, com $b_{0 j}$ variáveis aleatórias com distribuição normal, sendo que $E\left(b_{0 j}\right)=0, \operatorname{var}\left(b_{0 j}\right)=\sigma_{0}^{2}$ e $\operatorname{cov}\left(b_{0 j}, e_{i j}\right)=0$. Nesse caso, tem-se um modelo multinível que está ajustando retas paralelas, uma para cada unidade do nível 2.

Pode-se ainda considerar que o coeficiente $\beta_{1 j}$ seja aleatório, ou seja, $\beta_{1 j}=\beta_{1}+b_{1 j}$, $\operatorname{com} b_{1 j}$ tendo distribuição normal com média zero, variância $\sigma_{1}^{2}, \operatorname{cov}\left(b_{0 j}, b_{1 j}\right)=\sigma_{01}$ e ainda $\operatorname{cov}\left(b_{1 j}, e_{i j}\right)=0$. Neste caso, tem-se

$$
y_{i j}=\beta_{0}+b_{0 j}+\left(\beta_{1}+b_{1 j}\right) x_{i j}+e_{i j}
$$

Note que em (2.2) estão sendo associadas às unidades do nível 2 retas não paralelas. É importante ressaltar que o modelo acima é diferente do modelo de regressão usual, em que $y_{i j}=\beta_{0 j}+\beta_{1 j} x_{i j}$ e os coeficientes são fixos. O modelo com coeficientes fixos também ajusta uma reta para cada unidade do nível 2, mas os modelos devem ser utilizados em situações diferentes. Por exemplo, quando o objetivo do estudo é examinar e comparar um grupo específico de unidades do nível 2, então o modelo com coeficientes fixos é adequado. Contudo, quando se tem uma amostra aleatória de unidades do nível 2 e o objetivo é fazer inferência para a população de unidades do nível 2, o modelo hierárquico (com coeficientes aleatórios) deve ser utilizado.

Uma outra característica importante dos modelos hierárquicos, conforme já mencionado, é que eles incorporam a correlação entre unidades de um mesmo nível. De fato, 
considerando o modelo (2.2) e supondo $b_{0 j}, b_{1 j}$ e $e_{i j}$ sejam normalmente distribuídos com média zero, a covariância entre duas unidades do nível 1 pertencentes a diferentes unidades do nível 2 é dada por

$$
\begin{aligned}
\operatorname{cov}\left(y_{i j}, y_{i^{\prime} j^{\prime}}\right)= & \operatorname{cov}\left(\beta_{0}+b_{0 j}+\left(\beta_{1}+b_{1 j}\right) x_{i j}+e_{i j}\right. \\
& \left.\beta_{0}+b_{0 j^{\prime}}+\left(\beta_{1}+b_{1 j^{\prime}}\right) x_{i^{\prime} j^{\prime}}+e_{i^{\prime} j^{\prime}}\right) \\
= & 0
\end{aligned}
$$

para $j \neq j^{\prime}$.

Por outro lado, a covariância entre duas unidades do nível 1 pertencentes à mesma unidade do nível 2 é dada por:

$$
\begin{aligned}
\operatorname{cov}\left(y_{i j}, y_{i^{\prime} j}\right)= & \operatorname{cov}\left(\beta_{0}+b_{0 j}+\left(\beta_{1}+b_{1 j}\right) x_{i j}+e_{i j}, \beta_{0}+b_{0 j}+\right. \\
& \left.\left(\beta_{1}+b_{1 j}\right) x_{i^{\prime} j}+e_{i^{\prime} j}\right)= \\
= & \operatorname{cov}\left(b_{0 j}, b u_{0 j}\right)+x_{i^{\prime} j} \operatorname{cov}\left(b_{0 j}, b_{1 j}\right)+x_{i j} \operatorname{cov}\left(b_{0 j}, b_{1 j}\right)+ \\
& \operatorname{cov}\left(b_{1 j} x_{i j}, b_{1 j} x_{i^{\prime} j}\right)= \\
= & \sigma_{0}^{2}+\left(x_{i j}+x_{i^{\prime} j}\right) \sigma_{01}+x_{i j} x_{i^{\prime} j} \sigma_{1}^{2} .
\end{aligned}
$$

Mesmo supondo que os coeficientes aleatórios sejam independentes, isto é, $\sigma_{01}=0$, a covariância entre duas unidades do nível 1 pertencentes à mesma unidade do nível 2 é diferente de zero, sendo

$$
\operatorname{cov}\left(y_{i j}, y_{i^{\prime} j}\right)=\sigma_{0}^{2}+x_{i j} x_{i^{\prime} j} \sigma_{1}^{2}
$$

Os modelos hierárquicos ainda permitem que os coeficientes aleatórios possam depender de outras covariáveis, como

$$
\begin{aligned}
& \beta_{0 j}=\gamma_{00}+\gamma_{01} Z_{0 j}+b_{0 j}, \\
& \beta_{1 j}=\gamma_{10}+\gamma_{11} Z_{1 j}+b_{1 j},
\end{aligned}
$$


em que $Z_{0 j}$ e $Z_{1 j}$ são covariáveis definidas para as unidades do nível $2, b_{0 j}$ e $b_{1 j}$ são os erros das unidades do nível 2. Neste caso, o modelo pode ser escrito como

$$
y_{i j}=\gamma_{00}+\gamma_{01} Z_{0 j}+\left(\gamma_{10}+\gamma_{11} Z_{1 j}\right) x_{1 i j}+b_{0 j}+b_{1 j} x_{1 i j}
$$

Esse modelo é mais complicado e difícil de ser interpretado, pois existe o termo $Z_{1 j} x_{1 i j}$, que representa a interação entre os níveis. No entanto, pode ser útil quando se tem estruturas de covariância mais complicadas, como, por exemplo, quando a variância cresce à medida em que o valor de alguma covariável aumenta.

\subsection{Modelos Lineares Multiníveis Generalizados}

Os modelos lineares generalizados, considerados com detalhes em McCullagh \& Nelder (1989), são uma extensão dos modelos lineares, sendo que o leque de distribuições da variável resposta é ampliado para qualquer distribuição pertencente à família exponencial. Além disso, com essa abordagem, a relação entre o valor esperado da variável resposta e o preditor linear pode ser dada por meio de uma função não linear. Os modelos lineares multiníveis generalizados são uma extensão dos modelos lineares generalizados, em que se acrescentam coeficientes aleatórios ao preditor linear.

No caso dos modelos multiníveis em que se supõe distribuição normal, é fácil verificar que a inclusão dos coeficientes aleatórios incorpora a dependência entre as observações, o que não é tão imediato nos modelos multiníveis generalizados. Para calcular a covariância entre observações, é preciso escrever o modelo de maneira conveniente. Será utilizado como exemplo o caso da distribuição de Poisson, que é a de maior interesse, pois, conforme será visto, existe uma equivalência com a verossimilhança associada a alguns modelos comuns em análise de sobrevivência.

Considere, então, um modelo hierárquico com estrutura de dois níveis e seja $y_{i j}$ 
a variável resposta observada para a $i$-ésima unidade do nível 1 da $j$-ésima unidade do nível 2. Suponha que $y_{i j} \mid m_{i j}$ tem distribuição de Poisson com média $m_{i j}$. Uma maneira conveniente de escrever $y_{i j}$ em função de sua média $m_{i j}$, para este caso, é:

$$
y_{i j}=m_{i j}+\sqrt{m_{i j}} \varepsilon_{i j}
$$

em que $\varepsilon_{i j}$ é uma variável aleatória com $E\left(\varepsilon_{i j}\right)=0$ e $\operatorname{var}\left(\varepsilon_{i j}\right)=1$. Note que, neste caso, $E\left(y_{i j}\right)=\operatorname{var}\left(y_{i j}\right)=m_{i j}$, conforme esperado para a distribuição de Poisson. Para o cálculo da covariância entre observações, é preciso apenas o conhecimento da média e da variância de $\varepsilon_{i j}$; não são necessárias mais informações sobre a distribuição desses erros.

Suponha agora o preditor linear

$$
\eta_{i j}=\sum_{k=1}^{p} \beta_{k j} x_{k i j},
$$

$\operatorname{com} \beta_{k j}$ parâmetros desconhecidos e assuma que

$$
E\left(y_{i j}\right)=m_{i j}=f\left(\eta_{i j}\right),
$$

em que $f$ é uma função não linear do preditor linear $\eta_{i j}=\sum_{k=1}^{p} \beta_{k j} x_{k i j}$, conhecida como função de ligação. A exemplo da seção anterior, alguns coeficientes do preditor podem ser aleatórios. Assim, pode-se ter, por exemplo,

$$
\eta_{i j}=\beta_{0 j}+\beta_{1 j} x_{1 i j}+\sum_{k=2}^{p} \beta_{k} x_{k i j},
$$

com $\beta_{0 j}=\beta_{0}+b_{0 j}$ e $\beta_{1 j}=\beta_{1}+b_{1 j}$, em que $b_{0 j}$ e $b_{1 j}$ são variáveis aleatórias de média zero e $\operatorname{var}\left(b_{0 j}\right)=\sigma_{00}, \operatorname{var}\left(b_{1 j}\right)=\sigma_{11}$ e $\operatorname{cov}\left(b_{0 j}, b_{1 j}\right)=\sigma_{01}$. Em geral, supõe-se que a distribuição dos erros $b_{k j}$ seja normal, mesmo quando a distribuição da variável resposta não é normal. Existem alguns artigos que consideram outras distribuições para os efeitos aleatórios, como Lee \& Nelder (1996), que consideram, por exemplo, a distribuição gama para os efeitos aleatórios no caso da distribuição de Poisson. 
Considere um modelo hierárquico simples com função de ligação logarítmica, em que

$$
m_{i j}=\exp \left(\beta_{0}+\beta_{1} x_{i j}+b_{o j}\right)
$$

ou seja, apenas o intercepto é aleatório. Sejam $y_{i j}$ e $y_{i^{\prime} j^{\prime}}$ duas observações pertencentes a diferentes unidades do nível 2. A covariância entre essas observações é dada por:

$$
\operatorname{cov}\left(y_{i j}, y_{i^{\prime} j^{\prime}}\right)=\operatorname{cov}\left(m_{i j}+\sqrt{m_{i j}} \varepsilon_{i j}, m_{i^{\prime} j^{\prime}}+\sqrt{m_{i^{\prime} j^{\prime}}} \varepsilon_{i^{\prime} j^{\prime}}\right)=0
$$

uma vez que há independência dos erros $\varepsilon$ e dos termos $b_{0 j}$ e, assim, as médias $m_{i j}$ e $m_{i^{\prime} j^{\prime}}$ são independentes.

Considere, agora, duas observações $y_{i j}$ e $y_{i^{\prime} j}$ pertencentes à mesma unidade do nível 2. A covariância entre elas é dada por

$$
\begin{gathered}
\operatorname{cov}\left(y_{i j}, y_{i^{\prime} j}\right)=\operatorname{cov}\left(m_{i j}, m_{i^{\prime} j}\right)+\operatorname{cov}\left(m_{i j}, \sqrt{m_{i^{\prime} j}} \varepsilon_{i^{\prime} j}\right)+ \\
\operatorname{cov}\left(\sqrt{m_{i j}} \varepsilon_{i j}, m_{i^{\prime} j}\right)+\operatorname{cov}\left(\sqrt{m_{i j}} \varepsilon_{i j}, \sqrt{m_{i^{\prime} j}} \varepsilon_{i^{\prime} j}\right)
\end{gathered}
$$

Como os termos $\varepsilon_{i j}$ são independentes de $b_{0 j}$ e, portanto, das médias $m_{i j}$ e ainda $E\left(\varepsilon_{i j}\right)=0$, tem-se:

$$
\begin{gathered}
\operatorname{cov}\left(m_{i j}, \sqrt{m_{i^{\prime} j}} \varepsilon_{i^{\prime} j}\right)=E\left(m_{i j} \sqrt{m_{i^{\prime} j}} \varepsilon_{i^{\prime} j}\right)-E\left(m_{i j}\right) E\left(\sqrt{m_{i^{\prime} j}} \varepsilon_{i^{\prime} j}\right)= \\
=E\left(m_{i j} \sqrt{m_{i^{\prime} j}}\right) E\left(\varepsilon_{i^{\prime} j}\right)-E\left(m_{i j}\right) E\left(\sqrt{m_{i^{\prime} j}}\right) E\left(\varepsilon_{i^{\prime} j}\right)=0
\end{gathered}
$$

Assim, utilizando raciocínio análogo para os outros termos na expressão obtida para a $\operatorname{cov}\left(y_{i j}, y_{i^{\prime} j}\right)$, chega-se a

$$
\begin{aligned}
\operatorname{cov}\left(y_{i j}, y_{i^{\prime} j}\right) & =\operatorname{cov}\left(m_{i j}, m_{i^{\prime} j}\right)= \\
& =\operatorname{cov}\left(\exp \left(\beta_{0}+\beta_{1} x_{i j}+b_{o j}\right), \exp \left(\beta_{0}+\beta_{1} x_{i^{\prime} j}+b_{o j}\right)\right) \\
& =\exp \left(\beta_{0}+\beta_{1} x_{i j}+\beta_{0}+\beta_{1} x_{i j}\right) \operatorname{cov}\left(\exp \left(b_{0 j}\right), \exp \left(b_{0 j}\right)\right)
\end{aligned}
$$


Portanto,

$$
\begin{aligned}
\operatorname{cov}\left(y_{i j}, y_{i^{\prime} j}\right) & =\exp \left(2 \beta_{0}+\beta_{1}\left(x_{i j}+x_{i^{\prime} j}\right)\right) \operatorname{cov}\left(\exp \left(b_{0 j}\right), \exp \left(b_{0 j}\right)\right)= \\
& =\left[\exp \left(2 \beta_{0}+\beta_{1}\left(x_{i j}+x_{i^{\prime} j}\right)\right)\right]\left[E\left(\exp \left(2 b_{0 j}\right)\right)-\left[E\left(\exp \left(b_{0 j}\right)\right)\right]^{2}\right] .
\end{aligned}
$$

Como está assumido que a distribuição dos erros $b_{0 j}$ é normal com média zero e variância $\sigma_{0}^{2}$, então, utilizando os resultados da função geradora de momentos da distribuição normal, tem-se:

$$
\begin{aligned}
\operatorname{cov}\left(y_{i j}, y_{i^{\prime} j}\right) & =\left[\exp \left(2 \beta_{0}+\beta_{1}\left(x_{i j}+x_{i^{\prime} j}\right)\right)\right]\left[\exp \left(2 \sigma_{0}^{2}\right)-\exp \left(\sigma_{0}^{2}\right)\right]= \\
& =\left[\exp \left(2 \beta_{0}+\beta_{1}\left(x_{i j}+x_{i^{\prime} j}\right)\right)\right]\left(\exp \left(\sigma_{0}^{2}\right)-1\right) \exp \left(\sigma_{0}^{2}\right)
\end{aligned}
$$

Assim, a covariância é diferente de zero para observações pertencentes à mesma unidade do nível 2. É interessante observar que essa covariância é igual a zero se $\sigma_{0}^{2}=0$. De fato, se $\sigma_{0}^{2}=0$, então não há efeito das unidades do nível $2 \mathrm{e}$, portanto, as unidades do nível 1 pertencentes à mesma unidade do nível 2 são independentes. Se $\sigma_{0}^{2}$ é grande , então existe efeito da unidade do nível 2 e, dessa forma, a dependência entre observações pertencentes à mesma unidade do nível 2 deve ser grande.

Uma observação importante é que, no caso da distribuição de Poisson, muitas vezes deseja-se modelar a taxa de incidência $\pi_{i j}$ e não a média $m_{i j}$. Isso também pode ser feito no caso de modelos multiníveis. A relação entre a média e a taxa de incidência é dada por

$$
m_{i j}=M_{j} \pi_{i j}
$$

em que $M_{j}$ é uma constante conhecida. Fazendo $\pi_{i j}=\exp \left(\eta_{i j}\right)$, vem que

$$
\log \left(m_{i j}\right)=\log \left(M_{j}\right)+\eta_{i j}
$$

O termo $\log \left(M_{j}\right)$ é uma constante fixa, conhecida como offset. No processo de estimação, este termo é tratado como uma covariável com o coeficiente conhecido (igual a 1). Em 
particular, nos modelos de sobrevivência paramétricos, o offset será bastante utilizado. Conforme será visto posteriormente, esse termo é escolhido de maneira conveniente para que os resultados da distribuição de Poisson possam ser utilizados na estimação dos parâmetros do modelo de sobrevivência. 


\section{Capítulo 3}

\section{Modelos Multiníveis de riscos proporcionais}

\subsection{Introdução}

Em análise de sobrevivência, a variável resposta normalmente é o tempo até a ocorrência de um fenômeno. A estrutura hierárquica pode aparecer de várias maneiras diferentes em dados dessa natureza. Um exemplo é quando se tem repetições no mesmo indivíduo. Outra forma é quando se tem uma única observação em cada unidade amostral, que por sua vez são agrupados em unidades maiores. Um exemplo é o caso de estudos envolvendo membros de uma mesma família ou dentes de um mesmo indivíduo.

Uma característica muito comum que aparece em dados de análise de sobrevivência é a ocorrência de observações censuradas, quando se tem apenas informação parcial a respeito do tempo de ocorrência do evento de interesse, como por exemplo é o caso de saber-se que a falha ocorreu depois de um determinado instante ou em algum intervalo 
de tempo. No exemplo dos dentes citado acima, ao término do estudo pode não ter ocorrido a queda do selante de alguns dentes. Assim, não se tem conhecimento do tempo exato de queda do selante de alguns dentes, mas sabe-se que o tempo até a queda é maior do que um determinado valor (censura à direita). As técnicas mais difundidas em análise de sobrevivência procuram utilizar essa informação, apesar de incompleta. Para um revisão dos diversos tipos de censura, bem como de formas de lidar com o problema, ver, por exemplo, Cox \& Oakes (1984). Neste capítulo será apresentada uma generalização de algumas das técnicas mais conhecidas, para que elas incorporem efeitos aleatórios e, portanto, a dependência entre as observações. Inicialmente serão introduzidos a notação utilizada e alguns resultados já conhecidos e, depois, serão apresentados métodos de estimação e testes de hipóteses para modelos multiníveis.

\subsection{Modelos de riscos proporcionais}

Os modelos de riscos proporcionais formam a classe de modelos mais popular em análise de sobrevivência. Para iniciar a discussão dessa classe de modelos, denote por $T_{i j}$ a variável de interesse, isto é, o tempo até a observação de um evento de interesse. No exemplo dos selantes, $T_{i j}$ representaria o tempo até a ocorrência da queda do selante do $i$-ésimo dente da $j$-ésima criança. Conforme já explicado, em muitos casos, não se tem informação sobre o exato valor de $T_{i j}$, mas sabe-se que é maior do que um determinado valor $C_{i j}$ (possivelmente aleatório), denominado tempo de censura, o que caracteriza a censura à direita. Note que o tempo exato $T_{i j}$ será conhecido somente se $T_{i j} \leq C_{i j}$. Os dados podem ser representados pelos pares de variáveis $\left(Y_{i j}, \delta_{i j}\right)$ definido da seguinte maneira:

$$
\begin{aligned}
Y_{i j} & =\min \left(T_{i j}, C_{i j}\right) \\
\delta_{i j} & =I\left(T_{i j} \leq C_{i j}\right),
\end{aligned}
$$


em que $I\left(T_{i j} \leq C_{i j}\right)$ é a função indicador, ou seja, $\delta_{i j}=1$ se o tempo $T_{i j}$ foi observado e $\delta_{i j}=0$ se a observação foi censurada.

Supondo que os tempos de falha $T_{i j}$ são variáveis aleatórias não negativas com função de distribuição $F_{i j}(\cdot)$, tem-se a função de risco

$$
\alpha_{i j}\left(t \mid \mathbf{X}_{i j}\right)=\lim _{\Delta t \rightarrow 0} \frac{P\left(t \leq T_{i j}<t+\Delta t \mid T_{i j} \geq t, \mathbf{X}_{i j}\right)}{\Delta t}
$$

em que $\mathbf{X}_{i j}$ representa um vetor de $p$ covariáveis associado com a observação $T_{i j}$. A função de risco é conhecida também como taxa instantânea de falha e fornece informação sobre a intensidade com que a observação pode falhar, a cada instante $t \geq 0$.

Neste trabalho, serão tratados alguns modelos de riscos proporcionais, ou seja, modelos em que a função de risco pode ser escrita na forma

$$
\alpha_{i j}\left(t \mid \mathbf{X}_{i j}\right)=\alpha_{0}(t) c(\boldsymbol{\beta})
$$

em que $c(\cdot)$ é uma função conhecida, que não depende de $t$. Se uma distribuição contínua é especificada para $T_{i j}$, então $\alpha_{0}(t)$, denominada função de risco padrão, será uma função conhecida de $t$ e o modelo é dito paramétrico. Alternativamente, Cox (1972) apresenta um modelo em que $\alpha_{0}(t)$ é uma função arbitrária, que ficou conhecido como modelo de riscos proporcionais de Cox. Serão abordados neste trabalho extensões dos modelos de riscos proporcionais paramétricos (exponencial e Weibull) e o modelo semi-paramétrico de Cox para dados com estrutura hierárquica. Nos casos em que não existe uma estrutura hierárquica, o preditor linear $\eta_{i j}$ é formado por parâmetros fixos: $\eta_{i j}=\beta_{0}+\beta_{1} x_{1 i j}+$ $\ldots+\beta_{p} x_{p i j}$. Nos modelos com estrutura hierárquica, alguns dos coeficientes são tomados aleatórios, conforme já explicado.

O primeiro método de estimação que será apresentado posteriormente consiste basicamente em trabalhar com a densidade marginal dos dados. Em linha gerais, será utilizado o resultado $f_{X}(x)=\int f_{X, Y}(x, y) d y=\int f_{X \mid Y}(x \mid y) f_{Y}(y) d y$. Por esse motivo, torna-se importante trabalhar com a verossimilhança condicional aos efeitos aleatórios. O segundo 
método de estimação que será apresentado consiste basicamente em uma aproximação de Taylor e também utiliza resultados da verossimilhança condicional aos efeitos aleatórios. Nas seções 3.2.1 e 3.2.2, os resultados apresentados são condicionais aos efeitos aleatórios, uma vez que dados os coeficientes aleatórios, as observações são independentes. No desenvolvimento das seções, o condicionamento não será explicitado para simplificar a notação. Esses resultados já são bastante conhecidos para o caso em que se tem apenas coeficientes fixos e também são válidos considerando-se dados os efeitos aleatórios.

\subsubsection{Modelos paramétricos de riscos proporcionais}

Para simplificar a notação, considere a situação em que os dados apresentam uma estrutura hierárquica com dois níveis. A extensão para casos com mais de dois níveis é imediata. Seja $S_{i j}(t)$ a função de sobrevivência correspondente à i-ésima unidade do nível 1 da jésima unidade do nível 2 e $\alpha_{i j}(t)$ a função de risco. Serão considerados os modelos de riscos proporcionais em que $c(\boldsymbol{\beta})=\exp \left(\eta_{i j}\right)$ ou

$$
\alpha_{i j}\left(t \mid \mathbf{X}_{i j}\right)=\alpha_{0}(t) e^{\eta_{i j}}
$$

com $\eta_{i j}=\mathbf{X}_{i j} \boldsymbol{\beta}_{j}$. Relembrando, a matriz de coeficientes $\boldsymbol{\beta}_{\boldsymbol{j}}$ contém alguns coeficientes aleatórios no caso dos modelos hierárquicos. Assim, $\beta_{k j}=\beta_{k}+b_{k j}$ para alguns índices $k$, em que $b_{k j}$ são variáveis aleatórias com média zero e variância $\sigma_{k}^{2}$. Uma forma conveniente de escrever o preditor linear é $\eta_{i j}=\mathbf{X}_{i j} \boldsymbol{\beta}+\mathbf{Z}_{i j} \mathbf{b}$, em que $\boldsymbol{\beta}$ representa o vetor de coeficientes fixos, $\mathbf{b}$ representa o vetor de todos os efeitos aleatórios $b_{j k}$ e $\mathbf{Z}_{i j}$ é uma matriz conhecida, construída de forma adequada ao modelo. Nesta seção e na próxima, todo o desenvolvimento será feito considerando-se dado o vetor b de coeficientes aleatórios.

Uma importante relação entre a função de risco e a função de sobrevivência é que

$$
S_{i j}\left(t \mid \mathbf{X}_{i j}\right)=e^{-\int_{o}^{t} \alpha_{i j}\left(s \mid \mathbf{X}_{i j}\right) d s}
$$


Para o caso considerado em (3.1), tem-se que

$$
\begin{aligned}
S_{i j}\left(t \mid \mathbf{X}_{i j}\right) & =\exp \left(-\int_{0}^{t} \alpha_{0}(s) d s e^{\eta_{i j}}\right) \\
& =\exp \left(-\Lambda_{0}(t) e^{\eta_{i j}}\right),
\end{aligned}
$$

com $\Lambda_{0}(t)$ representando a função de risco padrão integrada (ou acumulada). Denotando por $f_{i j}$ a função densidade de probabilidade do indivíduo correspondente, como

$$
\alpha_{i j}\left(t \mid \mathbf{X}_{i j}\right)=\frac{F_{i j}^{\prime}\left(t \mid \mathbf{X}_{i j}\right)}{1-F_{i j}\left(t \mid \mathbf{X}_{i j}\right)}=\frac{f_{i j}\left(t \mid \mathbf{X}_{i j}\right)}{S_{i j}\left(t \mid \mathbf{X}_{i j}\right)}
$$

segue que

$$
\begin{aligned}
f_{i j}\left(t \mid \mathbf{X}_{i j}\right) & =\alpha_{i j}\left(t \mid \mathbf{X}_{i j}\right) S_{i j}\left(t \mid \mathbf{X}_{i j}\right) \\
& =\alpha_{0}(t) e^{\eta_{i j}} \exp \left(-\Lambda_{0}(t) e^{\eta_{i j}}\right) \\
& =\alpha_{0}(t) \exp \left(\eta_{i j}-\Lambda_{0}(t) e^{\eta_{i j}}\right)
\end{aligned}
$$

Considere, agora, a variável indicadora de censura $\delta_{i j}$ já definida anteriormente. A verossimilhança $L$ para dados com censura é dada por (para mais detalhes, ver Klein \& Moeschberger (1997)):

$$
L=\prod_{i, j}\left(f\left(t_{i j} \mid \mathbf{X}_{i j}\right)\right)^{\delta_{i j}}\left(S\left(t_{i j} \mid \mathbf{X}_{i j}\right)\right)^{1-\delta_{i j}},
$$

sendo que $\prod_{i, j}$ está sendo usado para representar um produtório duplo $\prod_{j=1}^{J} \prod_{i=1}^{n_{j}}$, em que $J$ é o número de unidades do nível 2 e $n_{j}$ é o número de unidades do nível 1 pertencentes à $j$-ésima unidade do nível 2. Esse produtório é simplesmente o produto de todas as observações do nível 1. Essa mesma convenção será utilizada no caso de somatórios.

Tomando-se o logaritmo da verossimilhança, encontra-se:

$$
l=\log L=\sum_{i, j}\left[\delta_{i j} \log \left(f\left(t_{i j} \mid \mathbf{X}_{i j}\right)\right)+\left(1-\delta_{i j}\right) \log \left(S\left(t_{i j} \mid \mathbf{X}_{i j}\right)\right)\right] .
$$


Substituindo $f_{i j}\left(t \mid \mathbf{X}_{i j}\right)$ e $S_{i j}\left(t \mid \mathbf{X}_{i j}\right)$ pelas expressões (3.2) e (3.3), obtém-se:

$$
\begin{aligned}
l & =\sum_{i, j}\left[\delta_{i j}\left(\log \alpha_{0}\left(t_{i j}\right)+\eta_{i j}-\Lambda_{0}\left(t_{i j}\right) e^{\eta_{i j}}\right)+\left(1-\delta_{i j}\right)\left(-\Lambda_{0}\left(t_{i j}\right) e^{\eta_{i j}}\right)\right]= \\
& =\sum_{i, j}\left[\delta_{i j}\left(\log \left(\alpha_{0}\left(t_{i j}\right)\right)+\eta_{i j}\right)-\Lambda_{0}\left(t_{i j}\right) e^{\eta_{i j}}\right]
\end{aligned}
$$

Somando e subtraindo o termo $\delta_{i j} \log \left(\Lambda_{0}\left(t_{i j}\right)\right)$ da expressão anterior, encontra-se:

$$
l=\sum_{i, j}\left[\delta_{i j}\left(\log \Lambda_{0}\left(t_{i j}\right)+\eta_{i j}\right)-\Lambda_{0}\left(t_{i j}\right) e^{\eta_{i j}}+\delta_{i j} \log \left(\frac{\alpha_{0}\left(t_{i j}\right)}{\Lambda_{0}\left(t_{i j}\right)}\right)\right]
$$

Fazendo $\mu_{i j}=\Lambda_{0}\left(t_{i j}\right) e^{\eta_{i j}}$, tem-se:

$$
l=\sum_{i, j}\left(\delta_{i j} \log \mu_{i j}-\mu_{i j}\right)+\sum_{i, j} \delta_{i j} \log \left(\frac{\alpha_{0}\left(t_{i j}\right)}{\Lambda_{0}\left(t_{i j}\right)}\right)
$$

O segundo termo da expressão (3.4) envolve apenas a função $\alpha_{0}(\cdot)$ e o indicador $\delta_{i j} \mathrm{e}$ não depende de $\eta_{i j}$, ou seja, não depende dos parâmetros a serem estimados. Além disso, é interessante comparar o primeiro termo do lado direito de (3.4) com o logaritmo da verossimilhança de variáveis aleatórias com distribuição de Poisson com média $\mu_{i j}$. Para isso, considere variáveis $w_{i j}$ independentes com distribuição de Poisson. A verossimilhança é

$$
L^{*}=\prod_{i, j} \frac{e^{\mu_{i j}} \mu_{i j}^{w_{i j}}}{w_{i j} !}
$$

e o logaritmo pode ser escrito na forma

$$
l^{*}=\log L^{*}=\sum_{i, j}\left(w_{i j} \log \mu_{i j}-\mu_{i j}\right)+\text { constante }
$$

A expressão (3.5) pode ser vista como sendo a verossimilhança de um modelo linear generalizado com distribuição de Poisson. Assim, é possível modelar a média $\mu_{i j}$ por meio da relação $\mu_{i j}=\Lambda_{0}\left(t_{i j}\right) e^{\eta_{i j}}$. Essa parametrização é conveniente, pois, utilizando a função 
logarítmica como função de ligação, obtém-se $\log \Lambda_{0}\left(t_{i j}\right)+\eta_{i j}$, ou seja, o termo $\log \Lambda_{0}\left(t_{i j}\right)$ exerce o papel de offset. Comparando-se as expressões (3.4) e (3.5), pode-se verificar que o núcleo do logaritmo da verossimilhança $l$ é igual ao núcleo da verossimilhança de variáveis aleatórias $w_{i j}$ com distribuição de Poisson. Assim sendo, pode-se estimar os parâmetros do modelo de riscos proporcionais utilizando-se procedimentos computacionais desenvolvidos para o modelo linear generalizado de Poisson. Deve-se apenas observar que o processo de estimação deve levar em conta que offset $\log \Lambda_{0}\left(t_{i j}\right)$, em geral, depende de parâmetro(s) desconhecido(s).

No caso da distribuição exponencial, a função de risco é dada por $\alpha\left(t_{i j} \mid \mathbf{X}_{i j}\right)=e^{\eta_{i j}}$. Logo, tem-se que

$$
\begin{gathered}
\alpha_{0}\left(t_{i j}\right)=1, \\
\Lambda_{0}\left(t_{i j}\right)=\int_{0}^{t_{i j}} 1 d s=t_{i j}
\end{gathered}
$$

e a densidade é

$$
f\left(t_{i j}\right)=\exp \left(\eta_{i j}-t_{i j} e^{\eta_{i j}}\right) .
$$

Assim, $\log \mu_{i j}=\log t_{i j}+\eta_{i j}$. Nota-se, dessa forma, que o offset é $\log t_{i j}$, que não depende de nenhum parâmetro desconhecido. Portanto, os parâmetros para o modelo exponencial podem ser estimados facilmente. Um caso um pouco mais geral é aquele em que $\alpha_{0}\left(t_{i j}\right)=$ $\alpha, \alpha>0$. Nesse caso, $\Lambda_{0}\left(t_{i j}\right)=\alpha t_{i j}$ e a densidade é dada por

$$
f\left(t_{i j}\right)=\alpha \exp \left(\eta_{i j}-\alpha t_{i j} e^{\eta_{i j}}\right) .
$$

Observe que a constante $\alpha$ pode ser escrita como $\alpha=\exp (a)$ e incorporada, então, no intercepto do preditor linear.

Para a distribuição Weibull, tem-se que a função de risco é dada por $\alpha_{i j}\left(t \mid \mathbf{X}_{i j}\right)=$ $\lambda t_{i j}^{\lambda-1} e^{\eta_{i j}}$. Logo,

$$
\alpha_{0}\left(t_{i j}\right)=\lambda t_{i j}^{\lambda-1}
$$


e

$$
\Lambda_{0}\left(t_{i j}\right)=t_{i j}^{\lambda}
$$

Assim, o segundo termo de (3.4) depende de $\alpha_{0}\left(t_{i j}\right) / \Lambda_{0}\left(t_{i j}\right)=\lambda / t_{i j}$. Portanto, o parâmetro $\lambda$ precisa ser estimado juntamente com os parâmetros do preditor linear e o processo de estimação deve ser modificado um pouco. Por (3.3), tem-se que a densidade neste caso fica dada por

$$
f\left(t_{i j}\right)=\lambda t_{i j}^{\lambda-1} \exp \left(\eta_{i j}-t_{i j}^{\lambda} e^{\eta_{i j}}\right)
$$

e, por (3.4), o logaritmo da verossimilhança é

$$
\begin{aligned}
l & =\sum_{i, j}\left(\delta_{i j} \log \mu_{i j}-\mu_{i j}\right)+\sum_{i, j} \delta_{i j}\left(\log \left(\frac{\lambda}{t_{i j}}\right)\right) \\
& =\sum_{i, j} \delta_{i j}\left(\log \lambda-\log t_{i j}\right)+\sum_{i, j}\left(\delta_{i j} \log \mu_{i j}-\mu_{i j}\right),
\end{aligned}
$$

$\operatorname{com} \mu_{i j}=t_{i j}^{\lambda} e^{\eta_{i j}}$

Para estimar os parâmetros nesse modelo, McCullagh \& Nelder (1989) sugerem encontrar a equação de estimação de $\lambda$ e acrescentar um passo ao processo de estimação. Derivando-se $l$ em relação à $\lambda$ e igualando a expressão a zero, obtém-se:

$$
\frac{\partial l}{\partial \lambda}=\sum_{i, j} \frac{\delta_{i j}}{\lambda}+\sum_{i, j}\left(\delta_{i j}-\mu_{i j}\right) \log t_{i j}=0
$$

Assim, a equação de estimação de $\lambda$ fica dada por:

$$
\hat{\lambda}=\frac{r}{\sum_{i, j}\left(\hat{\mu}_{i j}-\delta_{i j}\right) \log t_{i j}},
$$

em que $r$ é o número de observações não censuradas.

Para a estimação dos parâmetros no modelo exponencial, deve-se inicialmente criar variáveis auxiliares de Poisson para cada observação. Utilizando-se essas novas variáveis, obtém-se os valores numéricos das estimativas por meio de algum algoritmo desenvolvido 
para o modelo de Poisson com ligação logarítmica e com a presença de um offset, que é uma constante fixa conhecida, conforme já discutido no capítulo 2 . No caso do modelo Weibull, a estimação é iniciada com $\lambda=1$, ou seja, com a distribuição exponencial. Em seguida, estima-se o valor de $\lambda$ pela equação (3.6) e o processo iterativo é repetido com o valor de $\lambda$ atualizado até a convergência. Nas aplicações que serão apresentadas posteriormente, utilizou-se como critério de convergência a distância euclidiana entre os dois últimos valores encontrados para $\lambda$. Considerou-se que o algoritmo convergiu se a diferença entre os dois últimos valores, em módulo, era menor do que $10^{-6}$.

\subsubsection{Modelo semiparamétrico de Cox}

O modelo de riscos proporcionais de Cox é definido pela propriedade

$$
\alpha(t \mid \mathbf{X})=\alpha_{0}(t) \exp (\eta)
$$

em que $\mathbf{X}$ é um vetor dado de covariáveis e $\eta$ é o preditor linear. A principal diferença entre o modelo semiparamétrico de Cox e os modelos paramétricos discutidos anteriormente é que no modelo de Cox nenhuma estrutura é imposta à função de risco padrão $\alpha_{0}(t)$. Note que $\alpha_{0}(t)$ pode ser interpretado como sendo o risco médio de falha de indivíduos com todas as covariáveis iguais a zero.

Para estimar os parâmetros deste modelo, Cox (1972) propõe a utilização da verossimilhança parcial. Supondo que ocorra apenas uma falha em $t_{i^{\prime} j^{\prime}}$, a probabilidade condicional de que um indivíduo com covariáveis $\mathbf{X}_{i^{\prime} j^{\prime}}$ falhe neste instante, dado que um 
indivíduo pertencente ao grupo de risco $R\left(t_{i^{\prime} j^{\prime}}\right)$ falha, é dada por

$$
\begin{aligned}
& \frac{P\left[\text { indivíduo falhar em } t_{i^{\prime} j^{\prime}} \mid \text { sobreviveu até } t_{i^{\prime} j^{\prime}}\right]}{P\left[\text { uma falha em } t_{i^{\prime} j^{\prime}} \mid \text { sobreviveu até } t_{i^{\prime} j^{\prime}}\right]} \\
= & \frac{\alpha\left(t_{i^{\prime} j^{\prime}} \mid \mathbf{X}_{i^{\prime} j^{\prime}}\right)}{\sum_{R\left(t_{i^{\prime} j^{\prime}}\right)} \alpha\left(t_{i j} \mid \mathbf{X}_{i j}\right)}=\frac{\alpha_{0}\left(t_{i^{\prime} j^{\prime}}\right) \exp \left(\eta_{i^{\prime} j^{\prime}}\right)}{\sum_{R\left(t_{i^{\prime} j^{\prime}}\right)} \alpha_{0}\left(t_{i^{\prime} j^{\prime}}\right) \exp \left(\eta_{i j}\right)} \\
= & \frac{\exp \left(\eta_{i^{\prime} j^{\prime}}\right)}{\sum_{R\left(t_{i^{\prime} j^{\prime}}\right)} \exp \left(\eta_{i j}\right)}
\end{aligned}
$$

A verossimilhança parcial de Cox é obtida multiplicando-se as expressões (3.7) para todos os tempos em que alguma falha ocorreu:

$$
L=\prod_{\left(i^{\prime} j^{\prime}\right) \in F} \frac{\exp \left(\eta_{i^{\prime} j^{\prime}}\right)}{\sum_{R\left(t_{i^{\prime} j^{\prime}}\right)} \exp \left(\eta_{i j}\right)}
$$

em que $F$ representa o conjunto de indivíduos cujos tempos de falha são observados.

Para estimar os parâmetros, nesse caso também é possível encontrar uma equivalência com a verossimilhança de Poisson, mas as variáveis auxiliares devem ser criadas de maneira diferente. É possível obter também equivalência com a verossimilhança multinomial, conforme será mostrado, mas por conveniência computacional o processo de estimação será baseado no caso de Poisson. Considerando uma estrutura hierárquica de dois níveis, suponha que $t_{k}$ representa um tempo de falha e defina a variável indicadora $y_{i j(k)}$ da seguinte forma:

$$
y_{i j(k)}= \begin{cases}1 & \text { se falha } \\ 0 & \text { caso contrário }\end{cases}
$$

em que $i$ representa a unidade do nível $1, j$ a unidade do nível 2 e $(k)$ representa o tempo de falha para o qual o indivíduo (ij) pertence ao conjunto de risco. É importante ressaltar que essas variáveis $y_{i j(k)}$ são bastante diferentes dos indicadores de censura. Nesse caso, em todos os tempos de falha distintos, são criadas variáveis indicadoras para cada um dos indivíduos pertencentes ao grupo de risco. Assim, para cada observação serão criadas 
$n_{i j}$ variáveis indicadoras, com $n_{i j}$ representando o número de grupos de risco aos quais a observação pertence.

Para exemplificar, considere uma situação bem simples em que se tem apenas 5 indivíduos. Considere que o indivíduo $\mathrm{A}$ falhou em $t_{1}$, o indivíduo $\mathrm{B}$ foi censurado em $C_{1}$, o indivíduo $\mathrm{C}$ foi censurado em $C_{2}$, o indivíduo $\mathrm{D}$ falhou em $t_{2} \mathrm{e}$, finalmente, o indivíduo E foi censurado em $C_{3}$, com $t_{1} \leq C_{1} \leq C_{2}<t_{2} \leq C_{3}$. Temos, portanto, 2 tempos de falha distintos. Para o indivíduo A, seria apenas criada uma variável $y_{A(1)}=1$. Para os indivíduos $\mathrm{B}$ e C, teríamos também uma variável para cada: $y_{B(1)}=0$ e $y_{C(1)}=0$. Para o indivíduo D e E teríamos: $y_{D(1)}=0, y_{D(2)}=1, y_{E(1)}=0$ e, por fim, $y_{E(2)}=0$.

Para mostrar a equivalência com a verossimilhança de Poisson, considere as variáveis $y_{i j(k)}$ criadas em (3.8) e seja $\mu_{i j(k)}=\exp \left(\alpha_{k}+\eta_{i j}\right)$. Note que na expressão da média é introduzido o termo $\alpha_{k}$. Isso é um recurso utilizado para que se chegue à equivalência das verossimilhanças. Será suposto que as variáveis $y_{i j(k)}$ sejam independentes e que ocorra apenas uma falha em um determinado instante, ou seja, não há empates. Além disso, será suposto artificialmente que $y_{i j(k)}$ tenha distribuição de Poisson com média $\mu_{i j(k)}$. É claro que essas variáveis $y_{i j(k)}$ assumem apenas valores 0 ou 1 e, portanto, supor distribuição de Poisson não seria adequado. Contudo, será feita essa suposição artificial para que se chegue em uma expressão numérica equivalente à do modelo de Cox. Isso é apenas um recurso para se estimar os parâmetros no modelo semiparamétrico de Cox. Com essa suposição, então a verossimilhança para cada tempo de falha $t_{k}$, considerando que $o$ indivíduo $\left(i^{\prime} j^{\prime}\right)$ falhou neste instante, é dada por:

$$
\begin{aligned}
l_{k}^{*} & =\prod_{i j(a): a=k} \frac{e^{-\mu_{i j(k)}} \mu_{i j(k)}^{y_{i j(k)}}}{y_{i j(k)} !} \\
& =\frac{\exp \left(-\sum_{R} \mu_{i j(k)}\right) \exp \left(\alpha_{k}+\eta_{i^{\prime} j^{\prime}}\right)^{1}}{1 !}=\frac{\exp \left(\alpha_{k}+\eta_{i^{\prime} j^{\prime}}\right)}{\exp \left(\sum_{R} \exp \left(\alpha_{k}+\eta_{i j}\right)\right)} \\
& =\frac{e^{\alpha_{k}} \exp \left(\eta_{i^{\prime} j^{\prime}}\right)}{\exp \left(e^{\alpha_{k}} \sum_{R} \exp \left(\eta_{i j}\right)\right)}
\end{aligned}
$$


Como a soma de variáveis aleatórias com distribuição de Poisson tem a mesma distribuição com média igual à soma das médias, então $\sum_{R} y_{i j(k)}$ tem distribuição de Poisson com média $\mu_{S T}=\sum_{R} \mu_{i j(k)}$. Assim, tem-se que a estimativa de máxima verossimilhança de $\mu_{S T}$ é igual à soma dos $y_{i j(k)}=1$, uma vez que observou-se apenas uma falha. Portanto,

$$
\sum_{R} \mu_{i \hat{j}(k)}=e^{\hat{\hat{\alpha}_{k}}} \sum_{R} \exp \left(\eta_{i j}\right)=1 .
$$

Logo,

$$
e^{\hat{\alpha_{k}}}=\frac{1}{\sum_{R} \exp \left(\eta_{i j}\right)} .
$$

Assim, a verossimilhança para um tempo de falha é dada por:

$$
l_{k}^{*}=\frac{\frac{1}{\sum_{R} \exp \left(\eta_{i j}\right)} \exp \left(\eta_{i j}\right)}{\exp (1)}=\frac{\exp \left(\eta_{i j}\right)}{e \sum_{R} \exp \left(\eta_{i j}\right)} .
$$

Nota-se, portanto, que a verossimilhança $l_{k}^{*}$ dada em (3.9)é proporcional à expressão (3.7) . Assim, as estimativas do modelo de Cox coincidem com as do modelo de Poisson artificialmente criado (com exceção do intercepto). Esses resultados serão incorporados à estimação dos parâmetros na verossimilhança incondicional, conforme já discutido anteriormente.

Até agora foi tratado o caso em que não há empates. Quando isso ocorre, a aproximação sugerida por Breslow (1974) é utilizada. Suponha que sejam observadas $m$ falhas em um determinado instante e considere as variáveis auxiliares $y_{i j(k)}$ com distribuição de Poisson criadas anteriormente. A verossimilhança para estas variáveis no instante em que as $m$ observações falharam é dada por:

$$
\begin{aligned}
l_{k}^{*} & =\frac{\exp \left(-\sum_{R} \mu_{i j}\right) \exp \left(m \alpha_{k}+s\right)}{1 ! \ldots 1 !}=\frac{\exp \left(m \alpha_{k}+s\right)}{\exp \left(\sum_{R} \exp \left(\alpha_{k}+\eta_{i j}\right)\right)} \\
& =\frac{e^{m \alpha_{k}} \exp (s)}{\exp \left(e^{\alpha_{k}} \sum_{R} \exp \left(\eta_{i j}\right)\right)},
\end{aligned}
$$


em que $s=\sum_{D_{k}} \eta_{i j}$ e $D_{k}$ representa o conjunto dos índices $(i, j)$ dos indivíduos que falharam em $k$. Utilizando o mesmo raciocínio do caso em que não havia empates, podese concluir que a estimativa de máxima verossimilhança de $e^{\alpha_{k}}$ fica dada por:

$$
e^{\hat{\alpha_{k}}}=\frac{m}{\sum_{R} \exp \left(\eta_{i j}\right)}
$$

Substituindo essa expressão na verossimilhança (3.10) , chega-se ao resultado:

$$
l_{k}^{*}=\frac{\frac{m^{m}}{\left(\sum_{R} \exp \left(\eta_{i j}\right)\right)^{m} \exp (s)}}{\exp (m)}=\frac{m^{m}}{\exp (m)} \frac{\exp (s)}{\left(\sum_{R} \exp \left(\eta_{i j}\right)\right)^{m}} .
$$

Note que a verossimilhança obtida é proporcional à aproximação sugerida por Breslow (1974). Portanto, também pode-se utilizar os resultados obtidos para a distribuição de Poisson quando há empates.

Todo o processo de estimação dos modelos multiníveis será desenvolvido com base na equivalência com a verossimilhança de Poisson, mas é interessante observar que também há equivalência com a verossimilhança de uma distribuição multinomial. Considere, então, que $r_{i^{\prime} j^{\prime}}$ indivíduos estejam em risco no instante imediatamente anterior a $t_{i^{\prime} j^{\prime}}$ e ocorra apenas uma falha. Se a observação que falha for considerada como tendo distribuição multinomial com $r_{i^{\prime} j^{\prime}}$ categorias e probabilidade

$$
\pi_{i^{\prime} j^{\prime}}=\frac{e^{\eta_{i^{\prime} j^{\prime}}}}{\sum_{R} e^{\eta_{i j}}}
$$

então a contribuição para a verossimilhança é dada por:

$$
\frac{e^{\eta_{i^{\prime} j^{\prime}}}}{\sum_{R} e^{\eta_{i j}}}
$$

Portanto, as estimativas dos parâmetros do modelo multinomial acima serão iguais às do modelo de Cox. No entanto, esse resultado não será utilizado por conveniência computacional. 
Apesar de ter sido considerada a situação com apenas 2 níveis, a extensão a mais níveis pode ser feita facilmente. Para dados com estrutura hierárquica de três níveis, a única diferença com o desenvolvimento apresentado nesta seção seria a inclusão de mais um índice. Por exemplo, $t_{i j k}$ seria o tempo observado na $i$-ésima unidade do nível 1 , da $j$-ésima unidade do nível 2 da $k$-ésima unidade do nível 3.

\subsection{Inferência nos modelos Multiníveis}

Conforme mostrado na seção anterior, a estimação dos parâmetros dos modelos de sobrevivência multiníveis de riscos proporcionais pode ser feita utilizando-se resultados obtidos para o caso da distribuição de Poisson em um modelo linear generalizado misto. Assim sendo, nesta seção, serão apresentados métodos de estimação para modelos multiníveis generalizados, com distribuição de Poisson e, posteriormente, será abordada a questão de testes de hipóteses.

A característica fundamental dos modelos multiníveis é a existência de coeficientes aleatórios, o que dificulta muito a estimação dos parâmetros. Para utilização do método de máxima verossimilhança, por exemplo, baseada na distribuição marginal é preciso calcular integrais que, para problemas um pouco mais complexos, são intratáveis.

Para a estrutura hierárquica de dois níveis, com $y_{i j}$ tendo distribuição de Poisson com média $\mu_{i j}=e^{\eta_{i j}}$, suponha que $\eta_{i j}=\beta_{0 j}+\beta_{1 j} x_{1 i j}+\beta_{2 j} x_{2 i j}+\beta_{3} x_{3 i j}+\cdots+\beta_{p} x_{p i j}$, com, por exemplo, $\beta_{0 j}=\beta_{0}+b_{0 j}, \beta_{1 j}=\beta_{1}+b_{1 j}, \beta_{2 j}=\beta_{2}+b_{2 j}$, sendo $b_{0 j}, b_{1 j}$ e $b_{2 j}(j=1, \ldots, J)$ variáveis aleatórias independentes com distribuição normal com médias zero e variâncias $\sigma_{j}^{2}$. Pode-se, então, escrever

$\eta_{i j}^{b}=\beta_{0}+\beta_{1} x_{1 i j}+\beta_{2} x_{2 i j}+\beta_{3} x_{3 i j}+\cdots+\beta_{p} x_{p i j}+b_{0 j}+b_{1 j} x_{1 i j}+b_{2 j} x_{2 i j}$.

Será utilizada a notação $\eta_{i j}^{b}$ para indicar que o preditor linear depende dos coefici- 
entes aleatórios do vetor b. A expressão (3.11) pode ser reescrita em forma matricial

$$
\eta^{b}=\mathbf{X} \boldsymbol{\beta}+\mathbf{Z b}
$$

em que $\boldsymbol{\eta}^{b}$ é um vetor de $n$ linhas formado pelos elementos $\eta_{i j}^{b}, \boldsymbol{\beta}$ é um vetor de dimensão $p \times 1$ formado pelos coeficientes fixos $\beta_{0}, \ldots, \beta_{\boldsymbol{p}}, \mathbf{X}$ é uma matriz $n \times p$ conhecida, $\mathbf{Z}$ é uma matriz conhecida $n \times q$ e b é um vetor de $q$ linhas formado pelos coeficientes aleatórios $b_{01}, \ldots, b_{0 J}, b_{11}, \ldots, b_{1 J}, b_{21}, \ldots, b_{2 J}$. Note que a extensão para modelos hierárquicos com mais de dois níveis é imediata. A matriz de covariância de b, que será denotada por $\mathbf{D}$, será uma matriz bloco diagonal

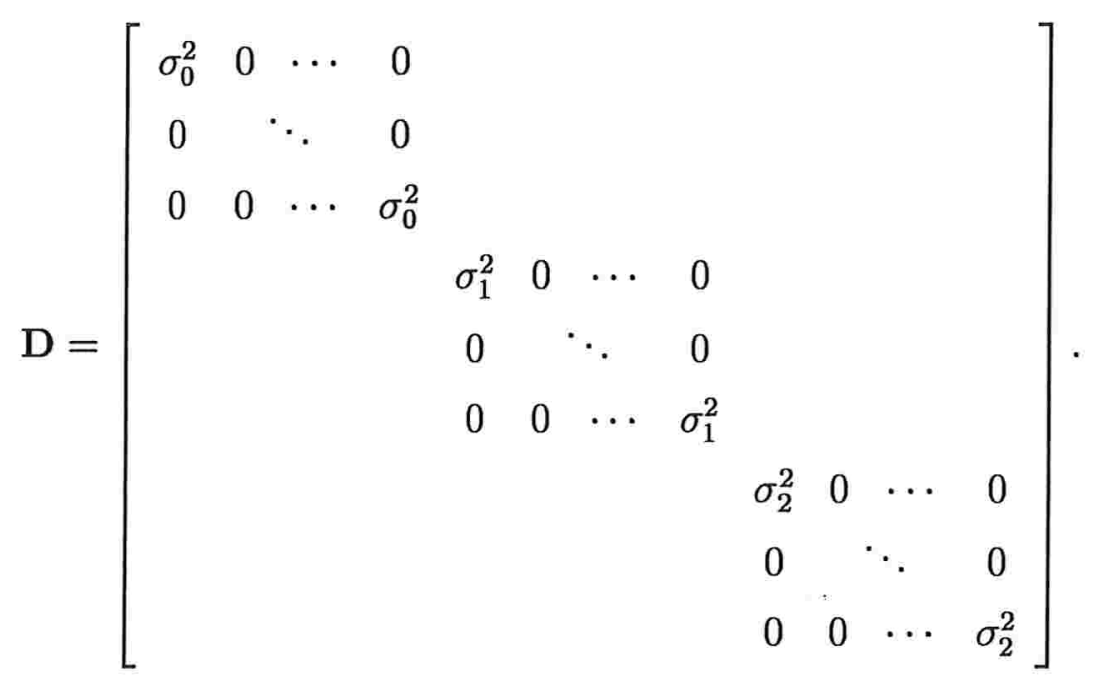

Denote por $\boldsymbol{\theta}$ o vetor formado pelos parâmetros da matriz de covariância $\mathbf{D}$. No exemplo, tem-se $\boldsymbol{\theta}=\left(\sigma_{0}^{2}, \sigma_{1}^{2}, \sigma_{2}^{2}\right)^{T}$. A verossimilhança marginal pode ser calculada integrando-se a densidade conjunta de y e b com relação a b. Assim, obtém-se

$$
L(\boldsymbol{\beta}, \boldsymbol{\theta})=\int f_{\mathbf{y}, \mathbf{b}}(\mathbf{y}, \mathbf{b}) d \mathbf{b}=\int f_{\mathbf{y} \mid \mathbf{b}}(\mathbf{y} \mid \mathbf{b}) f_{\mathbf{b}}(\mathbf{b}) d \mathbf{b}
$$

em que $f_{\mathbf{y} \mid \mathbf{b}}(\mathbf{y} \mid \mathbf{b})$ representa a densidade condicional das observações $y_{i j}$ dados os coeficientes aleatórios e $f_{\mathbf{b}}(\mathbf{b})$ representa a função densidade dos coeficientes aleatórios. 
Em particular, supondo que as observações $y_{i j}$ tenham distribuição de Poisson e sejam independentes dado o vetor $\mathbf{b}$ e, ainda, considerando-se que os coeficientes aleatórios possuem distribuição normal, a verossimilhança marginal fica dada por

$$
\begin{aligned}
L(\boldsymbol{\beta}, \boldsymbol{\theta}) & =\int \prod_{i, j} \frac{e^{-\mu_{i j}^{b}}\left(\mu_{i j}^{b}\right)^{y_{i j}}}{y_{i j} !} \times \frac{1}{(2 \pi)^{-k / 2}|\mathbf{D}|^{1 / 2}} e^{-\frac{1}{2} \mathbf{b}^{T} \mathbf{D}^{-1} \mathbf{b}} d \mathbf{b} \\
& \propto|\mathbf{D}|^{-1 / 2} \int \exp \left[\sum_{i, j} y_{i j} \log \mu_{i j}^{b}-\sum_{i, j} \mu_{i j}^{b}-\frac{1}{2} \mathbf{b}^{T} \mathbf{D}^{-1} \mathbf{b}\right] d \mathbf{b} .
\end{aligned}
$$

Note que $\mu_{i j}^{b}$ depende do vetor b de coeficientes aleatórios. Essa integral não é analiticamente calculável e, assim, deve ser utilizada uma aproximação para a obtenção de estimadores. Neste trabalho, serão discutidas basicamente duas formas de abordar o problema: a quase-verossimilhança penalizada (PQL) e a quase verossimilhança marginal (MQL), ambas discutidas em Breslow \& Clayton (1993).

\subsubsection{Quase Verossimilhança Penalizada}

Apesar do interesse ser a distribuição de Poisson neste trabalho, será apresentado aqui uma abordagem um pouco mais geral, conforme apresentada por Breslow \& Clayton (1993). Os autores desenvolvem o método de estimação para o caso em que se tem informação apenas sobre a esperança e variância das observações e, por isso, utilizam a abordagem da quase-verossimilhança. Para simplificar a notação, será utilizado apenas um índice para identificar cada observação. Supõe-se, então, que as variáveis $y_{i}$ sejam condicionalmente independentes dado o vetor $\mathbf{b}, \operatorname{com} \mathrm{E}\left(y_{i} \mid \mathbf{b}\right)=\mu_{i}^{b}$ e variância $\operatorname{var}\left(y_{i} \mid \mathbf{b}\right)=\phi a_{i} v\left(\mu_{i}^{b}\right)$, em que $v(\cdot)$ é a função de variância, $a_{i}$ é uma constante conhecida e $\phi$ é o parâmetro de dispersão (McCullagh \& Nelder 1989). A média condicional está relacionada ao preditor linear $\eta_{i}^{b}=\mathbf{x}_{i}^{t} \boldsymbol{\beta}+\mathbf{z}_{i}^{t} \mathbf{b}$ pela função de ligação $g\left(\mu_{i}^{b}\right)=\eta_{i}^{b}$, em que $\boldsymbol{\beta}$ é um vetor de $p$ linhas de efeitos fixos. Fazendo $h=g^{-1}$ e denotando por $\mathbf{X}$ a matriz cujas linhas são formadas 
por $\mathbf{x}_{i}^{t}$ e $\mathbf{Z}$ a matriz com linhas dadas por $\mathbf{z}_{i}^{t}$, então tem-se

$$
\mathrm{E}(\mathbf{y} \mid \mathbf{b})=\boldsymbol{\mu}^{b}=h\left(\eta^{b}\right)=h(\mathbf{X} \boldsymbol{\beta}+\mathbf{Z} \mathbf{b})
$$

Com base nessas suposições, pode-se definir a função de quase verossimilhança integrada, dada por

$$
e^{q l(\boldsymbol{\beta}, \boldsymbol{\theta})} \propto|\mathbf{D}|^{-1 / 2} \int \exp \left[-\frac{1}{2 \phi} \sum_{i=1}^{n} d_{i}\left(y_{i} ; \mu_{i}^{b}\right)-1 / 2 \mathbf{b}^{T} \mathbf{D}^{-1} \mathbf{b}\right] d \mathbf{b},
$$

em que $d_{i}(y, \mu)$ definido por

$$
d_{i}(y ; \mu)=-2 \int_{y}^{\mu} \frac{y-u}{a_{i} v(u)} d u
$$

é denominado deviance, que representa, nos modelos lineares generalizados, uma distância entre o logaritmo da verossimilhança do modelo saturado e do modelo sob investigação. No caso em que as observações apresentam distribuição pertencente à família exponencial, a expressão (3.14) é a verossimilhança dos dados.

Em particular, no caso de Poisson, temos $a_{i}=\phi=1$, para todo $i$, e $v\left(\mu_{i}^{b}\right)=\mu_{i}^{b}$. Assim, obtemos

$$
d_{i}\left(y_{i} ; \mu_{i}^{b}\right)=-2 \int_{y_{i}}^{\mu_{i}^{b}} \frac{y_{i}-u}{u} d u=-2\left(y_{i} \log \mu_{i}^{b}-\mu_{i}^{b}-y_{i} \log y_{i}+y_{i}\right)
$$

e

$$
e^{q l(\boldsymbol{\beta}, \boldsymbol{\theta})} \propto|\mathbf{D}|^{-1 / 2} \int \exp \left[\sum_{i} y_{i} \log \mu_{i}^{b}-\sum_{i} \mu_{i}^{b}-1 / 2 \mathbf{b}^{T} \mathbf{D}^{-1} \mathbf{b}\right] d \mathbf{b},
$$

que é proporcional à (3.13).

A idéia básica desse método de estimação é obter uma aproximação para a função de quase verossimilhança integrada (3.14) e maximizá-la em relação a $\boldsymbol{\beta}$ supondo-se $\boldsymbol{\theta}$ fixo, ou seja, fixados valores para os parâmetros da variância. Uma vez obtidos os valores estimados do vetor de parâmetros $\boldsymbol{\beta}$, substitui-se $\boldsymbol{\beta}$ por $\hat{\boldsymbol{\beta}}$ em (3.14), obtendo-se, dessa 
forma, uma verossimilhança perfilada. Em seguida, obtém-se uma aproximação para a verossimilhança perfilada e maximiza-se a expressão encontrada com relação a $\boldsymbol{\theta}$. O processo de estimação é feito iterativamente, alternando-se entre as equações de estimação dos parâmetros e dos componentes da variância, conforme será visto.

A aproximação utilizada para a função de quase verossimilhança integrada é a aproximação de Laplace, descrita detalhadamente em Barndorff-Nielsen \& Cox (1989). Escrevendo a expressão (3.14) na forma $c|D|^{-1 / 2} \int e^{-k(\mathbf{b})} d \mathbf{b}$, obtém-se a expansão de $-k(\mathbf{b})$ em torno de seu ponto máximo $\tilde{\mathrm{b}}$ antes da integração. Aplicando-se o resultado apresentado em Barndorff-Nielsen \& Cox (1989), chega-se facilmente a

$$
q l(\boldsymbol{\beta}, \boldsymbol{\theta}) \approx-\frac{1}{2} \log |\mathbf{D}|-\frac{1}{2} \log \left|\mathbf{k}^{\prime \prime}(\tilde{\mathbf{b}})\right|-\mathbf{k}(\tilde{\mathbf{b}}),
$$

$\operatorname{com} \tilde{\mathbf{b}}=\tilde{\mathbf{b}}(\boldsymbol{\beta}, \boldsymbol{\theta})$ representando a solução de $\mathbf{k}^{\prime}(\mathbf{b})=0$.

Desenvolvendo-se as expressões de $k(\mathbf{b}), k^{\prime}(\mathbf{b})$ e $k^{\prime \prime}(\mathbf{b})$, apresentadas no apêndice A, chega-se, então, a

$$
\begin{aligned}
q l(\boldsymbol{\beta}, \boldsymbol{\theta}) & \approx-\frac{1}{2} \log |\mathbf{D}|-\frac{1}{2} \log \left|\mathbf{Z}^{t} W \mathbf{Z}+\mathbf{D}^{-1}\right|-\mathbf{k}(\tilde{\mathbf{b}}) \\
& \approx-\frac{1}{2} \log \left|\mathbf{Z}^{t} \mathbf{W Z D}+I\right|-\mathbf{k}(\tilde{\mathbf{b}})
\end{aligned}
$$

com

$$
\mathbf{k}(\tilde{\mathbf{b}})=-\frac{1}{2 \phi} \sum_{i=1}^{n} d_{i}\left(y_{i}, \mu_{i}^{b}\right)-\frac{1}{2} \tilde{\mathbf{b}}^{t} \mathbf{D}^{-1} \tilde{\mathbf{b}},
$$

$\tilde{\mathbf{b}}$ representando a solução de $\mathbf{k}^{\prime}(\mathbf{b})=0$ e $\mathbf{W}=\operatorname{diag}\left[\left(\phi a_{i} v\left(\mu_{i}^{b}\right)\left(g^{\prime}\left(\mu_{i}^{b}\right)\right)^{2}\right)^{-1}\right]$.

Supondo que $\mathbf{W}$ varia pouco como função da média, escolhe-se $\boldsymbol{\beta}$ que maximiza o segundo termo de (3.17), ou seja, $\mathbf{k}(\tilde{\mathbf{b}})$. Assim, $(\hat{\boldsymbol{\beta}}, \tilde{\mathbf{b}})$ maximizam a quase-verossimilhança penalizada, dada por:

$$
-\frac{1}{2 \phi} \sum_{i=1}^{n} d_{i}\left(y_{i}, \mu_{i}^{b}\right)-\frac{1}{2} \mathbf{b}^{t} \mathbf{D}^{-1} \mathbf{b} .
$$


Note que a expressão (3.18) é a quase-verossimilhança menos um termo que depende de $\mathbf{b}$ e $\boldsymbol{\theta}$, ou seja, é a quase-verossimilhança penalizada pelo termo $\frac{1}{2} \mathbf{b}^{t} \mathbf{D}^{-1} \mathbf{b}$, devido ao fato de se ter coeficientes aleatórios no modelo. Derivando-se (3.18) com relação ao vetor de parâmetros fixos $\beta$ e com relação ao vetor de coeficientes aleatórios b, então chega-se às equações de estimação

$$
U_{1}=\sum_{i=1}^{n} \frac{\left(y_{i}-\mu_{i}^{b}\right) \mathbf{x}_{i}}{\phi a_{i} v\left(\mu_{i}^{b}\right) g^{\prime}\left(\mu_{i}^{b}\right)}=\mathbf{0}
$$

e

$$
U_{2}=\sum_{i=1}^{n} \frac{\left(y_{i}-\mu_{i}^{b}\right) \mathbf{z}_{i}}{\phi a_{i} v\left(\mu_{i}^{b}\right) g^{\prime}\left(\mu_{i}^{b}\right)}-\mathbf{D}^{-1} \mathbf{b}=\mathbf{0} .
$$

Portanto, as estimativas de $\boldsymbol{\beta}$ e b são obtidas resolvendo-se as equações acima. Uma observação importante é que como b é um vetor de coeficientes aleatórios, não é muito adequado utilizar o termo estimativa de b. Contudo, esse termo é muito utilizado na literatura e a justificativa para isso é apresentada por Harville (1977). Segundo ele, podese pensar que o vetor da variável resposta y observado é resultado de uma realização do vetor aleatório b. Uma vez ocorrido b, y é um resultado dessa realização. Assim, para aquele particular vetor $\mathbf{y}, \mathbf{b}$ pode ser considerado como um vetor de parâmetros, sendo que a única diferença é que se supõe algum conhecimento sobre a origem de b.

Em particular, para o caso da distribuição de Poisson com ligação canônica, ou seja, $\mu_{i}^{b}=\exp \left(\eta_{i}^{b}\right)$, tem-se, das expressões anteriores, que

$$
\mathbf{k}(\mathbf{b})=-\sum_{i=1}^{n}\left[y_{i} \log \left(\frac{y_{i}}{\mu_{i}^{b}}\right)-\left(y_{i}-\mu_{i}^{b}\right)\right]-\frac{1}{2} \mathbf{b}^{t} \mathbf{D}^{-1} \mathbf{b} .
$$

Derivando-se $\mathbf{k}(\mathbf{b})$, chega-se a:

$$
\mathbf{k}^{\prime}(\mathbf{b})=\sum_{i=1}^{n}\left(y_{i}-\mu_{i}^{b}\right) \mathbf{z}_{i}-\mathbf{D}^{-1} \mathbf{b}
$$

e

$$
\mathbf{k}^{\prime \prime}(\mathbf{b})=-\sum_{i=1}^{n} \mathbf{z}_{i} \mu_{i}^{b} \mathbf{z}_{i}^{t}-\mathbf{D}^{-1}
$$


Finalmente, as equações para este exemplo ficam dadas por

$$
\sum_{i=1}^{n}\left(y_{i}-\mu_{i}\right) \mathbf{x}_{i} \mu_{i}^{b}=0
$$

$\mathrm{e}$

$$
\sum_{i=1}^{n}\left(y_{i}-\mu_{i}^{b}\right) \mu_{i}^{b} \mathbf{z}_{i}=\mathbf{D}^{-1} \mathbf{b} .
$$

A solução do sistema de equações (3.19) e (3.20) pode ser obtida pelo método Scoring de Fisher. Escrevendo $U_{1}$ e $U_{2}$ em forma matricial encontramos

$$
U_{1}=\mathbf{X}^{T} \mathbf{W}_{M}(\mathbf{y}-\boldsymbol{\mu})
$$

e

$$
U_{2}=\mathbf{Z}^{T} \mathbf{W}_{M}(\mathbf{y}-\boldsymbol{\mu})-\mathbf{D}^{-1} \mathbf{b},
$$

em que $\mathbf{W}_{M}=\operatorname{diag}\left[\left(\phi a_{i} v\left(\mu_{i}^{b}\right) g^{\prime}\left(\mu_{i}^{b}\right)\right)^{-1}\right]$. Fazendo $\nu=\mathbf{D}^{-1} \mathbf{b}$ e derivando-se com relação a $\beta$ e a $\nu$, obtém-se:

$$
\frac{\partial U_{1}}{\partial \boldsymbol{\beta}}=-\mathbf{X}^{T} \mathbf{W}_{M}\left(\frac{\partial \boldsymbol{\mu}}{\partial \boldsymbol{\beta}}\right)+\mathbf{X}^{T} \frac{\partial \mathbf{W}_{M}}{\partial \boldsymbol{\mu}}(\mathbf{y}-\boldsymbol{\mu})
$$

$\mathrm{e}$

$$
\frac{\partial U_{1}}{\partial \boldsymbol{\nu}}=-\mathbf{X}^{T} \mathbf{W}_{M}\left(\frac{\partial \boldsymbol{\mu}}{\partial \boldsymbol{\nu}}\right)+\mathbf{X}^{T} \frac{\partial \mathbf{W}_{M}}{\partial \boldsymbol{\nu}}(\mathbf{y}-\boldsymbol{\mu})
$$

Derivando-se $U_{2}$ com relação aos vetores de parâmetros $\boldsymbol{\beta}$ e $\boldsymbol{\nu}$, tem-se:

$$
\begin{gathered}
\frac{\partial U_{2}}{\partial \boldsymbol{\beta}}=-\mathbf{Z}^{T} \mathbf{W}_{M}\left(\frac{\partial \boldsymbol{\mu}}{\partial \boldsymbol{\beta}}\right)+\mathbf{Z}^{T} \frac{\partial \mathbf{W}_{M}}{\partial \boldsymbol{\mu}}(\mathbf{y}-\boldsymbol{\mu}) \\
\frac{\partial U_{2}}{\partial \boldsymbol{\nu}}=-\mathbf{Z}^{T} \mathbf{W}_{M}\left(\frac{\partial \boldsymbol{\mu}}{\partial \nu}\right)+\mathbf{Z}^{T} \frac{\partial \mathbf{W}_{M}}{\partial \nu}(\mathbf{y}-\boldsymbol{\mu})-I .
\end{gathered}
$$


Como o termo $(\mathbf{y}-\boldsymbol{\mu})$ tem esperança igual a zero, obtemos:

$$
\begin{aligned}
& \mathrm{E}\left[\frac{\partial U_{1}}{\partial \boldsymbol{\beta}}\right]=-\mathbf{X}^{t} \mathbf{W X} \\
& \mathrm{E}\left[\frac{\partial U_{1}}{\partial \nu}\right]=-X^{t} W \mathbf{Z} \frac{\partial \mathbf{b}}{\partial \nu}=-\mathbf{X}^{t} \mathbf{W} \mathbf{Z D} \\
& \mathrm{E}\left[\frac{\partial U_{2}}{\partial \boldsymbol{\beta}}\right]=-\mathbf{Z}^{t} \mathbf{W X} \\
& \mathrm{E}\left[\frac{\partial U_{2}}{\partial \boldsymbol{\nu}}\right]=-\mathbf{Z}^{t} \mathbf{W} \mathbf{Z D}-I
\end{aligned}
$$

Em geral, o método Scoring de Fisher é dado pela equação $\beta^{(m+1)}=\beta^{(m)}+$ $\left[\mathrm{E}\left(-U^{\prime}\right)\right]^{-1} U\left(\beta^{m}\right)$. Assim, a solução pode ser encontrada através da solução iterativa do sistema:

$$
\begin{aligned}
{\left[\begin{array}{cc}
\mathbf{X}^{t} W \mathbf{X} & \mathbf{X}^{t} \mathbf{W Z D} \\
\mathbf{Z}^{t} W \mathbf{X} & \mathbf{Z}^{t} \mathbf{W Z D}+I
\end{array}\right] } & \left(\begin{array}{c}
\boldsymbol{\beta} \\
\nu
\end{array}\right)= \\
& \left(\begin{array}{c}
U_{1} \\
U_{2}
\end{array}\right)+\left[\begin{array}{cc}
\mathbf{X}^{t} W \mathbf{X} & \mathbf{X}^{t} \mathbf{W Z D} \\
\mathbf{Z}^{t} \mathbf{W X} & \mathbf{Z}^{t} \mathbf{W Z D}+I
\end{array}\right]\left(\begin{array}{l}
\boldsymbol{\beta} \\
\boldsymbol{\nu}
\end{array}\right)
\end{aligned}
$$

Denotando por $\mathbf{y}^{*}$ o vetor formado por $y_{i}^{*}=\eta_{i}^{b}+\left(y_{i}-\mu_{i}^{b} g^{\prime}\left(\mu_{i}^{b}\right)\right)$, então pode-se escrever:

$$
\left[\begin{array}{cc}
\mathbf{X}^{t} \mathbf{W X} & \mathbf{X}^{t} \mathbf{W Z D} \\
\mathbf{Z}^{t} \mathbf{W X} & \mathbf{Z}^{t} \mathbf{W Z D}+I
\end{array}\right]\left(\begin{array}{c}
\boldsymbol{\beta} \\
\nu
\end{array}\right)=\left[\begin{array}{c}
\mathbf{X}^{t} W \mathbf{W y}^{*} \\
\mathbf{Z}^{t} \mathbf{W} \mathbf{y}^{*}
\end{array}\right]
$$

Desenvolvendo o sistema, pode-se ainda escrever as equações da seguinte forma:

$$
\mathbf{X}^{t} \mathbf{V}^{-1} \mathbf{X} \beta=\mathbf{X}^{t} \mathbf{V}^{-1} \mathbf{y}^{*}
$$

e

$$
\hat{\mathbf{b}}=\mathbf{D} \hat{\boldsymbol{\nu}}=\mathrm{DZ}^{t} \mathbf{V}^{-1}\left(\mathbf{y}^{*}-\mathbf{X} \hat{\boldsymbol{\beta}}\right),
$$

$\operatorname{com} \mathbf{V}=\mathbf{W}^{-1}+\mathbf{Z D Z}^{t}$ 
Essas são as equações de estimação de $\boldsymbol{\beta}$ e b para valores fixados de $\boldsymbol{\theta}$. Para encontrar as equações de estimação do vetor de parâmetros da variância $\theta$, substitui-se na expressão (3.17) os valores maximizados de $\boldsymbol{\beta}$ e b, obtendo-se uma verossimilhança perfilada aproximada para inferência em $\boldsymbol{\theta}$. Ainda, com mais algumas aproximações, chega-se a equações de estimação já obtidas para o caso normal (Harville 1977) em termos do vetor $\mathbf{y}^{*}$ e das matrizes $\mathbf{D}$ e $W$, conforme descrito a seguir.

A primeira aproximação é a substituição da função desvio $\sum_{i} d_{i}\left(y_{i}, \mu_{i}^{b}\right)$ pela estatística de Pearson $\sum_{i}\left(y_{i}-\mu_{i}\right)^{2} /\left(a_{i} v\left(\mu_{i}^{b}\right)\right)$. Se, na expressão da função deviance (3.15), a função $v(u)$ for substituída por $v(\mu)$, então, resolvendo-se a integral, chega-se na estatística de Pearson. Ignorando-se a dependência de $\mathrm{W}$ em $\boldsymbol{\theta}$ e substituindo b pela expressão (3.22), obtém-se

$$
\begin{aligned}
q l_{1}(\hat{\boldsymbol{\beta}}(\boldsymbol{\theta}) ; \boldsymbol{\theta}) \approx & -\frac{1}{2} \log \left|\mathbf{Z}^{t} \mathbf{W} \mathbf{Z D}+I\right|-\frac{1}{2}\left(\mathbf{y}^{*}-\boldsymbol{\eta}^{b}\right)^{t} \mathbf{W}\left(\mathbf{y}^{*}-\eta^{b}\right) \\
& -\frac{1}{2}\left(\mathbf{y}^{*}-\mathbf{X} \boldsymbol{\beta}\right)^{t} \mathbf{V}^{-1} \mathbf{Z} \mathbf{D} \mathbf{Z}^{t} \mathbf{V}^{-1}\left(\mathbf{y}^{*}-\mathbf{X} \boldsymbol{\beta}\right)
\end{aligned}
$$

Utilizando os resultados $|\mathbf{V}|=\left|\mathbf{W}^{-1}\right|\left|I+\mathbf{Z}^{t} \mathbf{W} \mathbf{Z}^{t} \mathbf{D}\right|$ e $\mathbf{V}^{-1}\left(\mathbf{y}^{*}-\mathbf{X} \boldsymbol{\beta}\right)=\mathbf{W}\left(\mathbf{y}^{*}-\boldsymbol{\eta}^{b}\right)$, apresentados em Harville (1977), chega-se a

$$
q l_{1}(\hat{\boldsymbol{\beta}}(\boldsymbol{\theta}) ; \boldsymbol{\theta}) \approx-\frac{1}{2} \log |\mathbf{V}|-\frac{1}{2}\left(\mathbf{y}^{*}-\mathbf{X} \hat{\boldsymbol{\beta}}\right) \mathbf{V}^{-1}\left(\mathbf{y}^{*}-\mathbf{X} \hat{\boldsymbol{\beta}}\right)
$$

Nesse caso, pode-se utilizar o estimador de máxima verossimilhança restrita para incorporar a perda de graus de liberdade, que ocorre devido ao fato de estar sendo utilizado $\hat{\boldsymbol{\beta}}$ (e não $\boldsymbol{\beta}$ ). A diferença entre o estimador de máxima verossimilhança usual e o restrito é o acréscimo de um termo no logaritmo da verossimilhança no caso do restrito. Para maiores detalhes sobre estimadores de máxima verossimilhança restritos, ver Harville (1977). Assim, tem-se:

$$
q l_{1}(\hat{\boldsymbol{\beta}}(\boldsymbol{\theta}) ; \boldsymbol{\theta}) \approx-\frac{1}{2} \log |\mathrm{V}|-\frac{1}{2} \log \left|\mathbf{X}^{t} \mathbf{V}^{-1} \mathbf{X}\right|-\frac{1}{2}\left(\mathbf{y}^{*}-\mathbf{X} \hat{\boldsymbol{\beta}}\right) \mathrm{V}^{-1}\left(\mathbf{y}^{*}-\mathbf{X} \hat{\boldsymbol{\beta}}\right)
$$


Essa expressão é a mesma encontrada para o caso normal. Portanto, as derivadas dessa função coincidem com as obtidas quando se tem normalidade, que já são conhecidas e estão apresentadas em Harville (1977) e Graybill (1969). Definindo $\mathbf{P}=\mathbf{V}^{-1}$ $\mathbf{V}^{-1} \mathbf{X}\left(\mathbf{X}^{t} \mathbf{V}^{-1} \mathbf{X}\right)^{-1} \mathbf{X}^{t} \mathbf{V}^{-1}$ e derivando-se (3.23) com relação a cada componente de $\boldsymbol{\theta}$, obtém-se as equações:

$$
-\frac{1}{2}\left[\left(\mathbf{y}^{*}-\mathbf{X} \hat{\boldsymbol{\beta}}\right)^{t} \mathbf{V}^{-1} \frac{\partial \mathbf{V}}{\partial \theta_{j}} \mathbf{V}^{-1}\left(\mathbf{y}^{*}-\mathbf{X} \hat{\boldsymbol{\beta}}\right)-\operatorname{tr}\left(\mathbf{P} \frac{\partial \mathbf{V}}{\partial \theta_{j}}\right)\right]=0 .
$$

A derivada segunda de (3.23) fica dada por:

$$
\begin{aligned}
\frac{\partial^{2} q l_{1}(\hat{\boldsymbol{\beta}}(\boldsymbol{\theta}) ; \boldsymbol{\theta})}{\partial \theta_{i} \partial \theta_{k}}= & -\frac{1}{2} \operatorname{tr}\left[\mathbf{P}\left(\frac{\partial^{2} \mathbf{V}}{\partial \theta_{i} \partial \theta_{k}}-\frac{\partial \mathbf{V}}{\partial \theta_{i}} \mathbf{P} \frac{\partial \mathbf{V}}{\partial \theta_{k}}\right)\right] \\
& +\frac{1}{2}\left(\mathbf{y}^{*}-\mathbf{X} \hat{\boldsymbol{\beta}}\right)^{t} \mathbf{V}^{-1}\left[\frac{\partial^{2} \mathbf{V}}{\partial \theta_{i} \partial \theta_{k}}-2 \frac{\partial \mathbf{V}}{\partial \theta_{i}} \mathbf{P} \frac{\partial \mathbf{V}}{\partial \theta_{k}}\right] \mathbf{V}^{-1}\left(\mathbf{y}^{*}-\mathbf{X} \hat{\boldsymbol{\beta}}\right)
\end{aligned}
$$

A matriz de informação de Fisher apresenta componentes dados por:

$$
i_{j k}=\frac{1}{2} \operatorname{tr}\left(\mathbf{P} \frac{\partial \mathbf{V}}{\partial \theta_{j}} \mathbf{P} \frac{\partial \mathbf{V}}{\partial \theta_{k}}\right)
$$

É importante destacar que a derivação apresentada das equações de estimação da quase-verossimilhança penalizada deve ser vista como uma motivação heurística, pois utiliza várias aproximações sem justificativas formais (Breslow \& Clayton 1993).

\subsubsection{Quase Verossimilhança Marginal}

Esse método foi proposto inicialmente por Goldstein (1991), mas recebeu esse nome no artigo de Breslow \& Clayton (1993). Neste caso, também será apresentada uma abordagem mais geral, que envolve outras distribuições, apesar do interesse básico deste trabalho ser a distribuição de Poisson. A idéia do método é linearizar a função de ligação e aplicar a metodologia utilizada para os modelos lineares. 
O modelo marginal é especificado pela média marginal dada por:

$$
\mathrm{E}\left(y_{i}\right)=\mu_{i}=h\left(\mathbf{x}_{i}^{t} \beta\right) .
$$

Essa expressão pode ser vista como uma aproximação de primeira ordem do modelo. Escrevendo $y_{i}=\mu_{i}^{b}+\varepsilon_{i}, \operatorname{com} \operatorname{var}\left(\varepsilon_{i}\right)=\phi a_{i} v\left(\mu_{i}^{b}\right)$ e $\mu_{i}^{b}=h\left(\mathbf{x}_{i}^{t} \boldsymbol{\beta}+\mathbf{z}_{i}^{t} \mathbf{b}\right)$, então, obtendo a expansão de Taylor de $\mu_{i}^{b}$ em torno de $\mathbf{b}=\mathbf{0}$, tem-se:

$$
y_{i} \approx h\left(\mathbf{x}_{i}^{t} \boldsymbol{\beta}\right)+h^{\prime}\left(\mathbf{x}_{i}^{t} \boldsymbol{\beta}\right) \mathbf{z}_{i} \mathbf{b}+\varepsilon_{i} .
$$

Tomando-se a esperança desta expressão, chega-se a (3.26). A variância de $y_{i}$ pode ser aproximada por $\operatorname{var}\left(h\left(\mathbf{x}_{i}^{t} \boldsymbol{\beta}\right)+h^{\prime}\left(\mathbf{x}_{i}^{t} \boldsymbol{\beta}\right) \mathbf{z}_{i} \mathbf{b}+\varepsilon_{i}\right)$, que, com a suposição de independência de $\mathbf{b}$ e os erros $\varepsilon_{i}$, fica dada por:

$$
\operatorname{var}\left(y_{i}\right)=\left(h^{\prime}\left(\mathbf{x}_{i}^{t} \boldsymbol{\beta}\right)\right)^{2} \mathbf{z}_{i}^{t} \mathbf{D} \mathbf{z}_{i}+\phi a_{i} v\left(\mu_{i}\right) .
$$

Assim, a variância de y pode ser escrita:

$$
\operatorname{var}(\mathbf{y})=\mathbf{V}_{\mathbf{0}}+\boldsymbol{\Delta}^{-1} \mathbf{Z D Z}^{t} \boldsymbol{\Delta}^{-1},
$$

$\operatorname{com} \mathbf{V}_{0}=\operatorname{diag}\left(\phi a_{i} v\left(\mu_{i}\right)\right)$ e $\boldsymbol{\Delta}=\operatorname{diag}\left(g^{\prime}\left(\mu_{i}\right)\right)$.

Temos, então, expressões aproximadas para a média e para a variância de $\mathbf{y}$, que não dependem de $\mathbf{b}$. A variância de $\mathbf{y}$ depende de $\boldsymbol{\theta}$, mas considerando-se este vetor de parâmetros fixo, pode-se utilizar quase-verossimilhança para observações dependentes, conforme descrito em McCullagh \& Nelder (1989). Fixado $\boldsymbol{\theta}$, as equações de estimação de quase-verossimilhança para observações dependentes são dadas por

$$
\mathrm{U}(\boldsymbol{\beta})=\frac{\partial \boldsymbol{\mu}}{\partial \boldsymbol{\beta}^{t}} \operatorname{var}^{-1}(\mathbf{y})(\mathbf{y}-\boldsymbol{\mu}) .
$$

Essa função $U(\boldsymbol{\beta})$ apresenta propriedades que fazem com que ela se comporte como o logaritmo de uma verossimilhança, para $\boldsymbol{\theta}$ fixado (para maiores detalhes em quaseverossimilhança, ver McCullagh \& Nelder (1989)). Nesse problema específico, a equação 
fica dada por:

$$
\begin{aligned}
\mathrm{U}(\boldsymbol{\beta}, \boldsymbol{\theta}) & =\mathbf{X}^{t} \Delta^{-1}\left(\mathrm{~V}_{0}+\Delta^{-1} \mathbf{Z D Z}^{t} \Delta^{-1}\right)^{-1}(\mathrm{y}-\mu) \\
& =\mathbf{X}^{t}\left(\Delta \mathrm{V}_{0} \Delta+\mathbf{Z D Z}^{t}\right)^{-1} \Delta(\mathbf{y}-\mu)=0
\end{aligned}
$$

Derivando-se com relação a $\boldsymbol{\beta}$, obtém-se:

$$
\begin{aligned}
\frac{\partial \mathrm{U}(\boldsymbol{\beta}, \boldsymbol{\theta})}{\partial \boldsymbol{\beta}} & =-\mathrm{X}^{t}\left(\Delta \mathrm{V}_{0} \Delta+\mathrm{ZDZ}^{t}\right)^{-1} \Delta \Delta^{-1} \mathbf{X} \\
& =-\mathbf{X}^{t}\left(\Delta \mathrm{V}_{0} \Delta+\mathrm{ZDZ}^{t}\right)^{-1} \mathbf{X}
\end{aligned}
$$

Aplicando-se o método Scoring de Fisher, a seguinte equação é encontrada, supondo ainda $\boldsymbol{\theta}$ fixado:

$$
\mathbf{X}^{t} \mathbf{V}^{-1} \mathbf{X} \boldsymbol{\beta}=\mathbf{X}^{t} \mathbf{V}^{-1}(\Delta(\mathbf{y}-\mu)+\eta)
$$

As estimativas dos parâmetros referentes aos coeficientes aleatórios, ou seja, $\boldsymbol{\theta}$, são obtidas pela mesma equação da quase verossimilhança penalizada, ou seja,

$$
\hat{\mathbf{b}}=\mathbf{D} \hat{\boldsymbol{\nu}}=\mathbf{D} \mathbf{Z}^{t} \mathbf{V}^{-1}\left(\mathbf{y}^{*}-\mathbf{X} \hat{\boldsymbol{\beta}}\right),
$$

$\operatorname{com} \mathbf{V}=W^{-1}+\mathbf{Z D Z}^{t}$

Note que, na quase verossimilhança marginal, as equações de estimação não incorporam os termos aleatórios $\mathbf{z}_{i}^{t} \mathbf{b}$ no preditor linear, como acontece na quase verossimilhança penalizada.

Uma forma de se obter a equação de estimação do vetor de parâmetros da variância $\boldsymbol{\theta}$, sugerida por Breslow \& Clayton (1993), é utilizar a verossimilhança da distribuição normal, com a variância de $\mathbf{y}$ dada por var(y) $=\mathbf{V}_{0}+\boldsymbol{\Delta}^{-1} \mathbf{Z D Z}^{t} \boldsymbol{\Delta}^{-1}$, com $\mathrm{V}_{0}=\operatorname{diag}\left(\phi a_{i} v\left(\mu_{i}\right)\right)$ e $\Delta=\operatorname{diag}\left(g^{\prime}\left(\mu_{i}\right)\right)$. Com isso, chega-se à mesma expressão de $q l_{1}(\hat{\boldsymbol{\beta}}(\boldsymbol{\theta}) ; \boldsymbol{\theta})$ obtida na verossimilhança penalizada. Assim, nesse caso pode-se também 
utilizar a verossimilhança (3.23) para obter as estimativas de $\boldsymbol{\theta}$ e as equações de estimação são as mesmas obtidas anteriormente. A única diferença é que, no caso marginal, tanto o preditor linear $\boldsymbol{\eta}$ quanto a média $\boldsymbol{\mu}$ não incorporam os termos aleatórios.

Para iniciar o processo iterativo nos dois métodos apresentados, pode-se obter as estimativas de $\beta$ do modelo linear generalizado supondo observações independentes. É preciso também obter valores iniciais para os parâmetros da variância (vetor $\boldsymbol{\theta}$ ). Com esses valores iniciais de $\boldsymbol{\theta}$, é possível calcular o valor da matriz $\mathbf{D}$ e da matriz $\mathbf{V}$. Com isso, obtém-se novas estimativas dos parâmetros de $\boldsymbol{\beta}$ e b pelas equações (3.19) e (3.20) no caso da PQL. Quanto à MQL, obtém-se novas estimativas de $\boldsymbol{\beta}$ pela equação (3.27). Obtidas as novas estimativas do parâmetro $\boldsymbol{\beta}$ (e b, na PQL), utilizam-se (3.24) e (3.25) para obter um novo valor de $\boldsymbol{\theta}$. O processo é repetido até a convergência.

A utilização desses métodos de estimação para os modelos de sobrevivência descritos é simples, considerando-se os resultados apresentados na seção 3.2. Foi visto que a verossimilhança condicional dos modelos paramétricos e do modelo de Cox é equivalente à verossimilhança de variáveis aleatórias com distribuição de Poisson criadas artificialmente e de forma conveniente. Tanto o método PQL quanto o MQL fornecem estimativas para o modelo em que a distribuição condicional é de Poisson, ou seja, permite obter as estimativas para os parâmetros do modelo das variáveis de Poisson artificialmente criadas. Como a verossimilhança condicional dessas variáveis de Poisson é equivalente à do modelo de sobrevivência, então é claro que as estimativas do modelo de Poisson devem coincidir com as estimativas dos parâmetros do modelo de sobrevivência. Dessa forma, para a estimação dos parâmetros de um certo conjunto de dados de sobrevivência, basta criar para aqueles dados as variáveis de Poisson adequadas e aplicar o algoritmo da PQL ou MQL para essas variáveis criadas. 


\subsubsection{Correção de viés da quase verossimilhança penalizada}

O viés dos estimadores dos coeficientes de regressão e dos componentes da variância foram estudados inicialmente por Breslow \& Lin (1995) para o caso com intercepto aleatório apenas e, posteriormente, Lin \& Breslow (1996) estudaram para o caso mais geral. Aqui serão apresentados apenas a idéia usada na obtenção da correção e o algoritmo proposto pelos autores para obter as estimativas corrigidas. Para maiores detalhes, ver Lin \& Breslow (1996).

Considerando a função de quase verossimilhança integrada (3.14), obtém-se a aproximação de Solomon-Cox (Solomon \& Cox 1992). Essa aproximação para a integral é obtida expandindo-se $\sum_{i=1}^{n} \frac{1}{2 \phi} d_{i}\left(y_{i} ; \mu_{i}^{b}\right)=\sum_{i=1}^{n} l_{i}(\boldsymbol{\beta} ; \mathbf{b})$ em série de Taylor em torno do ponto $\mathbf{b}=\mathbf{0}$ antes da integração. A expansão até o termo quadrático produz a aproximação de primeira ordem de Solomon -Cox:

$$
\begin{aligned}
l_{S 1}= & -\frac{1}{2} \log \left|\mathbf{I}+\mathbf{Z}^{T} \mathbf{W}-{ }_{0} \mathbf{Z D}\right|+\sum_{i=1}^{n} l_{i}(\boldsymbol{\beta} ; \mathbf{0}) \\
& +\frac{1}{2} \mathbf{r}-{ }_{0}^{T} \mathbf{Z D}\left(\mathbf{I}+\mathbf{Z}^{T} \mathbf{W}-{ }_{0} \mathbf{Z D}\right)^{-1} \mathbf{Z}^{T} \mathbf{r}_{0}
\end{aligned}
$$

em que $\underline{\mathbf{r}}_{b}$ é um vetor de resíduos dados por $\left(y_{i}-\mu_{i}^{b}\right) / \phi a_{i}, \mathbf{W}_{b}$ é a matriz diagonal com elementos $v\left(\mu_{i}^{b}\right) / \phi a_{i}$ na diagonal e $\mathbf{r}_{0}$ e $\mathbf{W}_{0}$ são $\mathbf{r}_{b}$ e $\mathbf{W}_{b}$, respectivamente, obtidos quando $\mathbf{b}=\mathbf{0}$.

Lin \& Breslow (1996) mostram que a inclusão dos polinômios de terceira e quarta ordem fornecem a aproximação de segunda ordem de Solomon-Cox, dada por:

$$
l(\boldsymbol{\beta}, \boldsymbol{\theta})=l_{S 1}(\boldsymbol{\beta}, \boldsymbol{\theta})+\frac{1}{2} \boldsymbol{\theta}^{T}\left(\mathbf{1}_{23}+\mathbf{1}_{24}\right),
$$

em que $\mathbf{l}_{23}$ e $\mathbf{l}_{24}$ são matrizes $c \times c$ ( $c=$ número de coeficientes aleatórios) com elementos dados por:

$$
\mathbf{l}_{23}(j, k)=-\frac{1}{2}\left[\mathbf{1}^{T} \mathbf{Z}_{k}^{(2)^{T}} \mathbf{W}_{1} \mathbf{Z}_{j} \mathbf{Z} j^{T} \mathbf{r}_{0}+\mathbf{1}^{T} \mathbf{Z}_{j}^{(2)^{T}} \mathbf{W}_{1} \mathbf{Z}_{k} \mathbf{Z} k^{T} \mathbf{r}_{0}\right]
$$


e

$$
\mathbf{l}_{24}(j, k)=-\frac{1}{4} \mathbf{1}^{T} \mathbf{Z}_{j}^{(2)^{T}} \mathbf{W}_{2} \mathbf{Z}_{k} \mathbf{1}^{T}
$$

As matrizes $\mathrm{W}_{1}$ e $\mathrm{W}_{2}$ são matrizes diagonais com elementos dados por $v^{\prime}\left(\mu_{i}^{0}\right) v\left(\mu_{i}^{0}\right) / \phi a_{i}$ e $\left[v^{\prime \prime}\left(\mu_{i}^{0}\right)\left(v\left(\mu_{i}^{0}\right)\right)^{2}+\left(v^{\prime}\left(\mu_{i}^{0}\right)\right)^{2} v\left(\mu_{i}^{0}\right)\right] / \phi a_{i}$. Note que $\mathbf{W}_{1}=\mathbf{W}_{2}$ para o caso da distribuição de Poisson. As matrizes $\mathbf{Z}_{k}, k=1, \ldots, c$ são matrizes obtidas pela decomposição $\mathbf{Z b}=\mathbf{Z}_{1} \mathbf{b}_{1}+\cdots+\mathbf{Z}_{c} \mathbf{b} c$, em que $\mathbf{b} k=\left(b_{k 1}, \ldots, b_{k J}\right)^{T}$ é um vetor referente a um efeito aleatório, com média zero e variância $\sigma_{k}^{2} \mathbf{I}$. A notação $\mathbf{A}^{(2)}$ indica a matriz formada pelos elementos da matriz $\mathbf{A}$ ao quadrado, $\mathbf{A}^{(2)}=\left\{a_{i j}^{2}\right\}$, e $\mathbf{1}$ indica um vetor coluna formado por elementos todos iguais a 1 , de dimensão adequada para o produto das matrizes.

Obtendo-se as derivadas de $l(\boldsymbol{\beta}, \boldsymbol{\theta})$ em relação a $\boldsymbol{\theta}$ na expressão (3.29), Lin \& Breslow (1996) encontram a expansão de Taylor em torno de $\boldsymbol{\theta}=\mathbf{0}$, que fica dada por:

$$
\sum_{i=1}^{n} l_{i}(\beta ; 0)+\left(\mathbf{l}_{11}+\mathbf{l}_{12}\right)^{T} \boldsymbol{\theta}+\frac{1}{2} \boldsymbol{\theta}^{T}\left(\mathbf{l}_{21}+\mathbf{l}_{22}+\mathbf{l}_{23}+\mathbf{l}_{24}\right) \boldsymbol{\theta}
$$

com $\mathbf{l}_{11}$ e $\mathbf{l}_{12}$ vetores $c \times 1$ e $\mathbf{l}_{21}$ e $\mathbf{l}_{22}$ matrizes $c \times c$, cujos componentes são dados por:

$$
\begin{gathered}
\mathbf{l}_{11}(j)=\frac{1}{2} \mathbf{r}_{0}^{T} \mathbf{Z}_{j} \mathbf{Z}_{j}^{T} \mathbf{r}_{0}, \\
\mathbf{l}_{12}(j)=-\frac{1}{2} \operatorname{tr}\left(\mathbf{Z}_{j}^{T} \mathbf{W}_{0} \mathbf{Z}_{j}\right) . \\
\mathbf{l}_{21}(j, k)=-\mathbf{r}_{0}^{T} \mathbf{Z}_{j} \mathbf{Z}_{j}^{T} \mathbf{W}_{0} \mathbf{Z}_{k} \mathbf{Z}_{k}^{T} \mathbf{r}_{0}, \\
\mathbf{l}_{22}(j, k)=\frac{1}{2} \mathbf{1}^{T}\left(\mathbf{Z}_{j}^{T} \mathbf{W}_{0} \mathbf{Z}_{k}\right)^{(2)} \mathbf{1} .
\end{gathered}
$$

O viés dos estimadores dos coeficientes de regressão fixos foi estudado por meio da diferença entre as equações (3.30) e (3.18). Com base nessa diferença, Lin \& Breslow (1996) propõem o estimador da PQL com correção de primeira ordem :

$$
\hat{\boldsymbol{\beta}}_{C P}^{(1)}=\hat{\boldsymbol{\beta}}-\frac{1}{2}\left(\left.\left(\mathbf{X}^{T} \mathbf{W}_{0} \mathbf{X}\right)^{-1} \mathbf{X}^{T} \mathbf{W}_{1} \mathbf{Z}^{(2)} \mathbf{J}\right|_{\boldsymbol{\beta}=\hat{\boldsymbol{\beta}}}\right) \boldsymbol{\theta}
$$


em que $\mathbf{J}=\operatorname{diag}\left(\mathbf{1}_{q 1}, \ldots, \mathbf{1}_{q c}\right)$.

A correção de segunda ordem é dada por:

$$
\begin{gathered}
\hat{\boldsymbol{\beta}}_{C P}^{(2)}=\quad \hat{\boldsymbol{\beta}}-\frac{1}{2}\left(\mathbf{X}^{T} \mathbf{W}_{0} \mathbf{X}\right)^{-1} \\
\times\left[\mathbf{X}^{T} \mathbf{W}_{1} \mathbf{Z}^{(2)} \mathbf{J} \boldsymbol{\theta}-\sum_{j=1}^{c} \sum_{k=1}^{c} \mathbf{X}^{T} \mathbf{W}_{1}\right. \\
\left.\quad\left(\left(\mathbf{Z}_{j} \mathbf{Z}_{j}^{T} \mathbf{W}_{0} \mathbf{Z}_{k}\right) \cdot \mathbf{Z}_{k}\right) \mathbf{1} \theta_{j} \theta_{k}\right]\left.\right|_{\boldsymbol{\beta}=\hat{\boldsymbol{\beta}}},
\end{gathered}
$$

em que o produto $\mathbf{G} \cdot \mathbf{H}$ representa o produto de Hadamard, ou seja, cada elemnto da matriz resultante é dado por $\left(g_{i j} h_{i j}\right)$. A correção do viés das estimativas dos parâmetros da variância (vetor $\boldsymbol{\theta}$ ) é feita comparando-se a derivada da verossimilhança perfilada obtida por meio da expressão (3.30) com a expressão aproximada dada em (3.23). O estimador de $\boldsymbol{\theta}$ corrigido fica dado por:

$$
\hat{\boldsymbol{\theta}}_{C P}=\mathbf{C}^{-1} \mathbf{C}_{P} \hat{\boldsymbol{\theta}}
$$

com

$$
\begin{gathered}
\mathbf{C}=\frac{1}{2} \mathbf{J}^{T}\left(\mathbf{Z}^{T} \mathbf{W}_{0} \mathbf{Z}\right)^{(2)} \mathbf{J}+\frac{1}{4} \mathbf{J}^{T} \mathbf{Z}^{(2)^{T}} \mathbf{W}_{2} \mathbf{Z}^{(2)} \mathbf{J}-\mathbf{B}^{T}\left(\mathbf{X}^{T} \mathbf{W}_{0} \mathbf{X}\right)^{-1} \mathbf{B}, \\
\mathbf{B}=\frac{1}{2} \mathbf{X}^{T} \mathbf{W}_{1} \mathbf{Z}^{(2)} \mathbf{J}
\end{gathered}
$$

$\mathrm{e}$

$$
\mathbf{C}_{P}=\frac{1}{2} \mathbf{J}^{T}\left(\mathbf{Z}^{T} \mathbf{W}_{0} \mathbf{Z}\right)^{(2)} \mathbf{J}
$$

Com isso, Lin \& Breslow (1996) propõem um algoritmo simples para obter as estimativas corrigidas dos parâmetros:

1. Obter as estimativas de $\boldsymbol{\beta}$ e $\boldsymbol{\theta}$ pelo método PQL (que serão denotadas por $\hat{\boldsymbol{\beta}}_{P}$ e $\hat{\boldsymbol{\theta}}_{P}$ ), bem como os respectivos erros padrões.

2. Corrigir $\hat{\boldsymbol{\theta}}_{P}$ por $\hat{\boldsymbol{\theta}}_{C P}=\mathbf{C}^{-1} \mathbf{C}_{P} \hat{\boldsymbol{\theta}}$. A estimativa da matriz de covariância é aproximada por $\mathbf{G} \operatorname{cov}\left(\hat{\boldsymbol{\theta}}_{P}\right) \mathbf{G}^{T}, \operatorname{com} \mathbf{G}=\mathbf{C}^{-1} \mathbf{C}_{P}$. 
3. Use $\hat{\boldsymbol{\theta}}_{C P}$ para estimar $\boldsymbol{\beta}$ novamente pelas equações de estimação para $\boldsymbol{\beta}$ da PQL. Denote essas novas estimativas por $\hat{\boldsymbol{\beta}}_{C P}^{(0)}$.

4. Corrija $\hat{\beta}_{C P}^{(0)}$ por

$$
\hat{\boldsymbol{\beta}}_{C P}^{(1)}=\hat{\boldsymbol{\beta}}_{C P}^{(0)}-\left(\mathbf{X}^{T} \mathbf{W}^{0} \mathbf{X}\right)^{-1} \mathbf{B} \hat{\boldsymbol{\theta}}_{C P}
$$

e

$$
\hat{\boldsymbol{\beta}}_{C P}^{(2)}=\hat{\boldsymbol{\beta}}_{C P}^{(1)}+\left(\mathbf{X}^{T} \mathbf{W}^{0} \mathbf{X}\right)^{-1} \mathbf{A}
$$

com

$$
\mathbf{B}=\frac{1}{2} \mathbf{X}^{T} \mathbf{W}_{1} \mathbf{Z}^{(2)} \mathbf{J}
$$

$\mathrm{e}$

$$
\mathbf{A}=\frac{1}{2} \sum_{j=1}^{c} \sum_{k=1}^{c} \mathbf{X}^{T} \mathbf{W}_{1}\left(\left(\mathbf{Z}_{j} \mathbf{Z}_{j}^{T} \mathbf{W}_{0} \mathbf{Z}_{k}\right) \cdot \mathbf{Z}_{k}\right) \mathbf{1} \hat{\boldsymbol{\theta}}_{C P}(j) \hat{\boldsymbol{\theta}}_{C P}(k) .
$$

As covariâncias das estimativas corrigidas ficam dadas por $\operatorname{cov}\left(\hat{\boldsymbol{\beta}}_{C P}^{(0)}\right)+$ $\left(\mathbf{X}^{T} \mathbf{W}^{0} \mathbf{X}\right)^{-1} \mathbf{B} \operatorname{cov}\left(\hat{\boldsymbol{\theta}}_{C P}\right) \mathbf{B}^{T}\left(\mathbf{X}^{T} \mathbf{W}^{0} \mathbf{X}\right)^{-1}$.

Conforme já dito, foram apresentados apenas os resultados obtidos por Lin \& Breslow (1996), mas como a implementação computacional deste algoritmo é simples, foram calculadas as estimativas corrigidas para os exemplos analisados no capítulo 4 .

\subsubsection{Testes de hipóteses - aplicação do método Bootstrap}

Uma vez obtidas as estimativas dos parâmetros, um teste para verificar se a variância dos efeitos aleatórios são iguais a zero é de bastante interesse nos modelos multiníveis. Se tais parâmetros são iguais a zero, então pode-se prosseguir a análise com a abordagem usual para dados independentes.

Como foram obtidas estimativas dos parâmetros da variâncias e estimativas para os respectivos erros padrões, pode-se pensar em fazer o teste de Wald. Contudo, até este 
momento, não há qualquer resultado formal com relação à distribuição assintótica dessa estatística quando se utiliza os métodos PQL ou MQL para estimar os parâmetros da variância. Uma forma de abordar esse problema é utilizar o método bootstrap (Efron \& Tibshirani 1993), que pode também ser utilizado para construir intervalos de confiança.

A idéia básica do método bootstrap é obter amostras da função de distribuição empírica. Para melhor entender o processo, considere uma amostra aleatória formada por $n$ elementos $x_{1}, \ldots, x_{n}$ e denote por $\hat{F}$ a função de distribuição empírica, que associa probabilidade $1 / n$ para cada um dos valores observados $x_{i}$. Uma amostra bootstrap é uma amostra aleatória de tamanho $n$ retirada da distribuição empírica $\hat{F}$. Em outras palavras, uma amostra bootstrap, que será denotada por $\left(x_{1}^{*}, \ldots, x_{n}^{*}\right)$, é uma amostra aleatória retirada com reposição do conjunto de dados $x_{1}, \ldots, x_{n}$. Em linhas gerais, o método consiste em retirar $B$ amostras bootstrap e, para cada uma dessas amostras, calcular a estatística de interesse $s\left(x_{1}^{*}, \ldots, x_{n}^{*}\right)$. Dessa forma, obtém-se $B$ valores de $s$ e, com base nesta distribuição empírica da estatística $s$, são construídos testes de hipóteses, correções de viés, intervalos de confiança, etc.

No caso de modelos multiníveis, a aplicação do bootstrap não é imediata, pois não se tem observações independentes. Assim, o método de reamostragem deve incorporar a estrutura hierárquica dos dados.

A reamostragem nos modelos multiníveis pode ser feita de várias maneiras. Considere um conjunto de dados com estrutura de dois níveis, em que existem $J$ unidades do nível 2. Uma possibilidade é fazer a reamostragem em ambos os níveis. Para isso, retira-se uma amostra com reposição de tamanho $J$ das unidades do nível 2, obtendo-se a amostra $j_{k}^{*}, k=1, \ldots, J$. Cada unidade do nível $2, j_{k}^{*}$, contém $I_{j_{k}}^{*}$ unidades do nível 1. Retira-se, então, uma amostra com reposição dessas $I_{j_{k}}^{*}$ unidades do nível 1. Esse procedimento fornece uma amostra bootstrap e, portanto, deve ser repetido $B$ vezes. Uma outra forma é fazer a reamostragem apenas nas unidades do nível 2. Assim, retiram-se amostras de 
tamanho $J$ de unidades do nível 2 e, para cada uma dessas unidades, são utilizadas as unidades do nível 1 existentes no conjunto inicial de dados, sem qualquer modificação. É possível também fixar as unidades do nível 2 e fazer a reamostragem apenas com as unidades do nível 1 dentro de cada unidade desse nível.

A forma como a reamostragem deve ser feita nos modelos multiníveis depende da natureza dos dados. Assim, quando se tem conjuntos de dados com medidas repetidas, em que as unidades do nível 2 são os indivíduos, em geral, é mais conveniente fazer a reamostragem nas unidades do nível 2 apenas. Se, por exemplo, as unidades do nível 2 estão sendo comparadas (como países), então é mais adequado fazer a reamostragem dentro das unidades do nível dois.

Para construir intervalos de confiança, será utilizado o método bootstrap-t (Efron \& Tibshirani 1993). Nesse método, a idéia é construir o intervalo de confiança utilizando-se os quantis de uma distribuição empírica obtida para aquele conjunto de dados e não com base nos quantis de alguma distribuição conhecida.

Considere que tenham sido retiradas $B$ amostras bootstrap do conjunto de dados. Para cada amostra, calcula-se o valor de

$$
t^{*}(b)=\frac{\hat{\kappa}^{* b}-\hat{\kappa}}{\sqrt{\operatorname{vâr}^{* b}}}
$$

em que vâr ${ }^{* b}$ é a estimativa da variância de $\hat{\kappa}^{* b}$ da amostra bootstrap b. Obtém-se, dessa forma, $B$ valores $t^{*}(b)$ e o percentil de ordem $\alpha$ desta distribuição é o valor $\hat{t}^{(\alpha)}$ tal que

$$
\frac{\#\left\{t^{*}(b) \leq \hat{t}^{(\alpha)}\right\}}{B}=\alpha \text {. }
$$

Com isso, o intervalo de confiança com coeficiente $\alpha$ é dado por:

$$
\left(\hat{\kappa}-\hat{t}^{(1-\alpha / 2)} \sqrt{\operatorname{vâr}(\hat{\kappa})} ; \hat{\kappa}-\hat{t}^{(\alpha / 2)} \sqrt{\operatorname{vâr}(\hat{\kappa})}\right) \text {. }
$$


Para fazer o teste de hipóteses, utiliza-se a mesma idéia do intervalo de confiança, isto é, constrói-se a distribuição empírica da estatística de teste com base nos dados. O valor da estatística de teste obtido na amostra é comparado com a distribuição empírica construída para se tomar uma decisão.

Suponha que o interesse esteja em verificar se $\mathrm{H}_{0}: \kappa=\kappa_{0}$. Para testar essa hipótese, seria necessário utilizar a distribuição da estatística do teste de Wald:

$$
w=\left(\hat{\kappa}-\kappa_{0}\right)^{2} / \operatorname{var}(\hat{\kappa})
$$

O problema é que, conforme já discutido, mesmo a distribuição assintótica dessa estatística não é conhecida. Se calcularmos $w$ para todas as amostras bootstrap, obtemos a distribuição empírica de $w$. Contudo, não é a distribuição sob $\mathrm{H}_{0}$. Para obtê-la, calculase a estatística

$$
\left.w^{* b}=\left(\hat{\kappa}^{* b}-\hat{\kappa}\right)^{2}\right) / \operatorname{var}\left(\hat{\kappa}^{b}\right)
$$

A idéia utilizada é transladar a distribuição empírica, para que ela fique em torno do valor zero, como é de se esperar sob a hipótese nula. Como a distribuição dos valores $\hat{k}^{* b}$ está em torno de $\hat{k}$, então $\hat{k}^{* b}-\hat{k}$ fica em torno do zero. Se a hipótese nula é falsa, a distribuição de $w$ deve estar em torno de algum valor diferente de zero. Assim, se a estatística $w$ se desviar muito de zero, deve-se rejeitar a hipótese nula. O nível descritivo do teste é obtido comparando-se o valor $\hat{w}$ calculado para a estatística $w$ com a distribuição empírica de $w^{* b}$ :

$$
\text { nível descritivo }=\frac{\#\left\{w^{* b}>\hat{w}\right\}}{B} \text {. }
$$

Neste trabalho, estaremos apenas considerando o método bootstrap não paramétrico, mas outras abordagens poderiam ser também utilizadas, como o método bootstrap paramétrico, discutido em Efron \& Tibshirani (1993). 


\section{Capítulo 4}

\section{Aplicações}

\subsection{Introdução}

A aplicação da metodologia descrita foi feita utilizando-se o aplicativo SAS, versão 8. No caso de modelos lineares generalizados mistos, as estimativas dos parâmetros feitas com base na quase verossimilhança marginal e na quase verossimilhança penalizada podem ser facilmente obtidas por meio da macro GLIMMIX.

Para a estimação nos modelos de sobrevivência, foram desenvolvidas macros que criam as variáveis auxiliares de Poisson convenientes, da maneira descrita no Capítulo 3. As macros foram desenvolvidas para o caso dos modelos paramétricos (exponencial e Weibull) e para o caso do modelo semiparamétrico de Cox e criam as variáveis auxiliares de Poisson adequadas ao modelo considerado. Um novo conjunto de dados com essas informações é então utilizado na macro GLIMMIX para se obter as estimativas de interesse. No caso do modelo Weibull, foi necessário também criar uma outra macro para estimar o parâmetro $\lambda$ através da equação (3.6). Para obter as estimativas com a 
correção do viés da quase verossimilhança penalizada, foi desenvolvido um programa que utiliza o módulo IML do SAS. As macros desenvolvidas podem ser obtidas no endereço ftp://ftp.ime.usp.br/pub/acarlos/multinivel.

No caso dos modelos de sobrevivência, os algoritmos parecem ser um pouco instáveis. Em geral, o método da quase verossimilhança marginal apresenta maiores problemas de convergência do que o método da quase verossimilhança penalizada. No caso da MQL, são necessárias mais iterações até atingir a convergência e, além disso, algumas vezes o algoritmo da MQL não converge, enquanto o da PQL o faz.

Os programas foram aplicados a três conjuntos de dados, que estão apresentados nas seções seguintes. Todo o processamento foi feito em um pentium II de $450 \mathrm{MHZ}$, com 160 MB de memória RAM.

\subsection{Comparação de selantes}

O objetivo do estudo era comparar dois produtos no uso como selantes, Delton e Optibond. Foram utilizadas 37 crianças, sendo 23 meninas e 15 meninos. Em uma mesma criança, metade dos dentes recebeu a aplicação do selante Delton e a outra metade, do adesivo OptiBond. A variável resposta era o tempo até a queda (parcial ou total) dos selantes. No total, são 171 observações, sendo observadas 43 falhas e 128 censuras $(74,8 \%$ das observações).

A análise foi feita utilizando-se um modelo simples, apenas com a covariável tratamento, que foi categorizada da seguinte forma:

$$
\text { Tratamento }= \begin{cases}1 & \text { se Optibond } \\ 0 & \text { se Delton }\end{cases}
$$


O modelo utilizado é, então, dado por:

$$
\alpha_{i j}\left(t \mid x_{i j}\right)=\alpha_{0}(t) e^{\eta_{i j}}
$$

com $\eta_{i j}=x_{i j} \beta_{j}=x_{i j}\left(\beta+b_{j}\right)$. Nesse caso, o índice $i j$ representa o $i$-ésimo dente (unidade do nível 1) da $j$-ésima criança (unidade do nível 2). Assume-se que as variáveis $b_{j}(j=$ $1, \ldots, 37)$ são independentes e apresentam distribuição normal com média zero e variância $\sigma^{2}$. A Tabela 4.1 apresenta os resultados obtidos.

Pode-se verificar que o método PQL fornece resultado próximo ao do modelo de independência. A estimativa da MQL também está próxima do modelo de independência e as demais apresentam valores um pouco menores. Os níveis descritivos dos testes de Wald utilizando o bootstrap foram obtidos com base em 1000 replicações bootstrap. Podese observar que o nível descritivo do teste $\beta=0$ supondo distribuição de qui-quadrado e o obtido pelo bootstrap apresentam resultados bem próximos. Foram também construídos intervalos de confiança pelo bootstrap para o parâmetro $\beta$. No caso da PQL, o intervalo com $95 \%$ de confiança obtido foi $(-1,2123 ;-0,5145)$ e no caso da MQL o intervalo foi $(-1,6729 ;-0,6150)$.

Observando os parâmetros da variância, pode-se verificar que as estimativas da PQL corrigidas são iguais à obtida sem a correção. Conforme explicado no capítulo 4, a correção do viés do parâmetro $\theta$ é obtida multiplicando-se o vetor estimado via PQL por uma matriz G. Nos exemplos estudados, verificou-se que a matriz G estava muito próxima da matriz identidade. Em todos os casos, o teste de Wald para o parâmetro da variância fornece fortes evidências de que não há dependência estatisticamente significante entre as observações para esse conjunto de dados. Além disso, o intervalo de $95 \%$ de confiança obtidos pelo bootstrap foi $(-0,2452 ; 0,6538)$ no caso da PQL, que inclui o zero. Dessa forma, o modelo de independência mostra-se adequado nessa situação. No caso da MQL, não foi possível calcular o intervalo de confiança, pois em muitas amostras bootstrap a estimativa de $\sigma^{2}$ obtida foi igual a zero e o erro padrão não foi calculado. Assim, não foi 


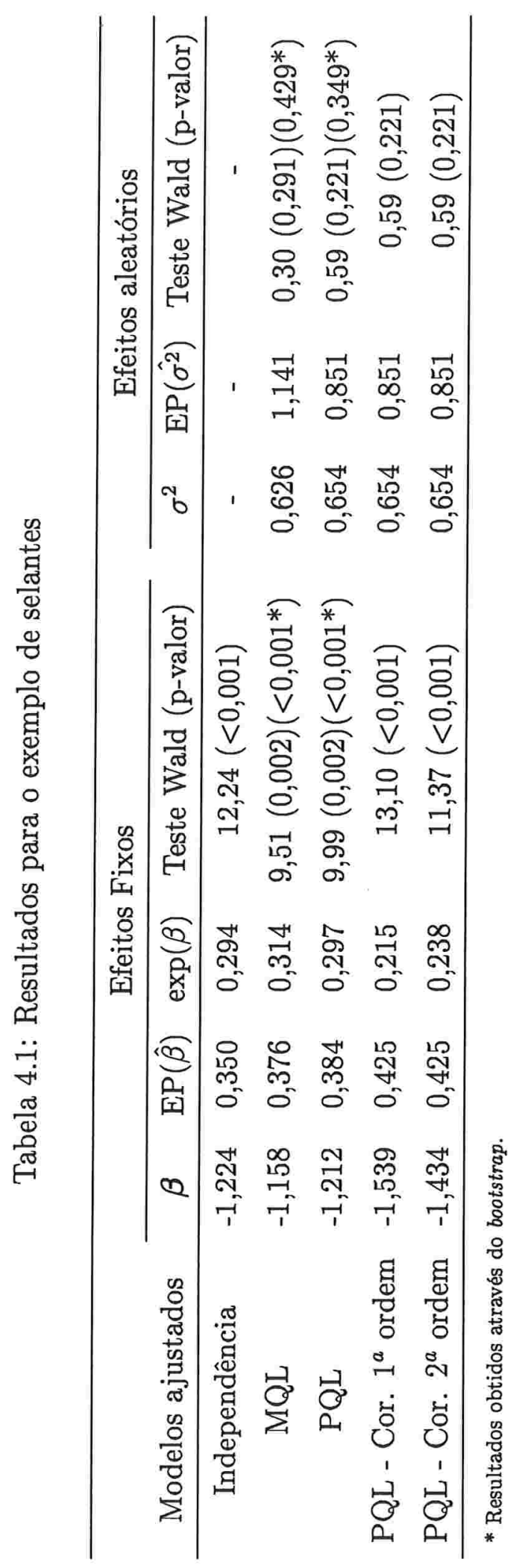


possível calcular a o valor de $\hat{t}^{\alpha / 2}$ descrito no capítulo anterior.

Uma observação interessante é com relação a resultados obtidos para esse mesmo conjunto de dados utilizando o modelo de fragilidade gama, apresentado em Chicarino (1999). O modelo de fragilidade gama é diferente dos estudados aqui, pois o efeito aleatório tem distribuição gama. Ainda assim, os resultados obtidos para os efeitos fixos estão próximos, levando às mesmas conclusões do ponto de vista prático. Por exemplo, Chicarino (1999) obteve como estimativa de $\beta$ o valor $-1,396$, com desvio padrão de 0,3707 (pelo método de estimação proposto por Nielsen). O teste para verificar se o modelo de independência adequado fornceceu p-valor igual a 0,102.

Para ilustrar os modelos paramétricos, foi feito o ajuste desses dados utilizando os modelos exponencial e Weibull. Em ambos os casos, considerou-se apenas a covariável tratamento e um intercepto aleatório. Assim, utilizou-se o preditor linear $\eta_{i j}=\alpha_{j}+\beta x_{i j}=$ $\alpha+b_{j}+\beta x_{i j}$, em que $b_{j}$ são variáveis aleatórias independentes com distribuição normal de média zero e variância $\sigma^{2}$. A covariável $x_{i j}$ representa o tratamento ( 1 se OptiBond e 0 se Delton) do i-ésimo dente da j-ésima criança. A Tabela 4.2 apresenta os resultados do ajuste exponencial e a Tabela 4.3 do modelo Weibull.

Assim como ocorreu no modelo semiparamétrico de Cox, no caso exponencial , o teste indica que o modelo de independência é adequado. No caso do modelo Weibull, o método MQL não convergiu em 50 iterações e por isso não é apresentado na tabela 4.3. No método PQL, o teste para verificar se o modelo de independência é adequado forneceu um resultado um pouco diferente. O p-valor obtido é igual a 2,3\%, que leva rejeição da hipótese nula. Um problema que pode estar ocorrendo é a falta de adequabilidade do modelo. Seria interessante, como trabalho futuro, desenvolver técnicas para verificar se o modelo ajustado é adequado aos dados.

É interessante observar que a estimativa do parâmetro $\beta$ nos modelos paramétricos 
Tabela 4.2: Resultados do ajuste exponencial para o exemplo de selantes

\begin{tabular}{|c|c|c|c|}
\hline \multirow[b]{2}{*}{ Parâmetros } & \multicolumn{3}{|c|}{ Modelos } \\
\hline & Independência & Multinível - MQL & Multinível - PQL \\
\hline$\alpha$ & $-3,805$ & $-3,680$ & $-3,741$ \\
\hline $\operatorname{EP}(\hat{\alpha})$ & 0,177 & 0,209 & 0,217 \\
\hline Teste Wald (p-valor) & $463,19(<0,001)$ & $310,46(<0,001)$ & $297,56(<0,001)$ \\
\hline$\beta$ & $-1,216$ & $-1,292$ & $-1,299$ \\
\hline $\operatorname{EP}(\hat{\beta})$ & 0,349 & 0,342 & 0,354 \\
\hline Teste Wald (p-valor) & $12,11(0,0005)$ & $14,29(<0,001)$ & $13,47(<0,001)$ \\
\hline$\sigma^{2}$ & - & 0,414 & 0,431 \\
\hline $\mathrm{EP}\left(\hat{\sigma^{2}}\right)$ & - & 0,446 & 0,322 \\
\hline Teste Wald (p-valor) & - & $0,86(0,352)$ & $1,79(0,180)$ \\
\hline
\end{tabular}

está muito próxima à obtida no modelo de Cox. Com relação aos testes de hipóteses nos casos paramétricos, também não há resultados teóricos formais com relação à estatística de Wald e, portanto, o uso do bootstrap também foi considerado. Contudo, no caso do modelo Weibull, não foi possível rodar o programa para o bootstrap, pois em determinado momento ocorria um erro e o programa era interrompido. Um teste do escore para o caso da distribuição Weibull pode ser desenvolvido com base no artigo de Lin (1997) e está sendo trabalhado por uma aluna de Doutorado do IME-USP. 
Tabela 4.3: Resultados do ajuste do modelo Weibull para o exemplo de selantes

\begin{tabular}{|c|c|c|}
\hline \multirow[b]{2}{*}{ Parâmetros } & \multicolumn{2}{|c|}{ Modelos } \\
\hline & Independência & Multinível - PQL \\
\hline$\alpha$ & $-5,83$ & $-6,338$ \\
\hline $\operatorname{EP}(\hat{\alpha})$ & 0,177 & 0,251 \\
\hline Teste Wald (p-valor) & $1089(<0,001)$ & $635,54(<0,001)$ \\
\hline$\beta$ & $-1,257$ & $-1,514$ \\
\hline $\operatorname{EP}(\hat{\beta})$ & 0,349 & 0,369 \\
\hline Teste Wald (p-valor) & $12,96(<0,001)$ & $16,81(<0,001)$ \\
\hline$\sigma^{2}$ & - & 0,886 \\
\hline $\operatorname{EP}\left(\hat{\sigma}^{2}\right)$ & - & 0,443 \\
\hline Teste Wald (p-valor) & - & $4,00(0,023)$ \\
\hline
\end{tabular}

A estimativa de $\lambda$ (expr. (3.6)) obtida no modelo de independência é 1,683 e no modelo multinível é 1,902.

\subsection{Doença granulomatosa crônica da infância}

Esta aplicação refere-se a um experimento com pacientes portadores da doença granulomatosa crônica da infância, caracterizada por um problema no sistema imunológico. As células dos pacientes são capazes de executar a fagocitose, mas a capacidade de matar os microrganismos ingeridos é falha. Isso acarreta infecções recorrentes graves que podem causar até a morte, geralmente na infância (para maiores detalhes sobre a doença, ver Zitelli \& Holly (1992) ). O objetivo do experimento era verificar se a incidência de infecções nos pacientes é reduzida com uso de gamma interferon.

O conjunto de dados consiste de 128 pacientes, dos quais 65 aleatoriamente receberam placebo e 63, gamma interferon. Os dados e sua descrição mais detalhada podem ser encontradas em Fleming \& Harrington (1991). A variável resposta desse estudo é o tempo até a ocorrência de infecção. Como um paciente pode apresentar mais de uma 
infecção durante o período em que o estudo foi realizado, então tem-se eventos recorrentes.

Esse conjunto de dados apresenta, de certa forma, uma estrutura hierárquica: cada paciente constitui uma unidade do nível 2 e suas infecções representam as unidades do nível 1. É importante ficar claro que, nesse caso, a variável resposta é o tempo até a ocorrência da infecção desde a ocorrência anterior ou início do tratamento (no caso da primeira infecção).

Neste trabalho, foi ajustado um modelo simples, contendo apenas a variável tratamento, codificada da seguinte maneira:

$$
\text { Tratamento }=\left\{\begin{array}{l}
1 \text { se placebo } \\
0 \text { se gamma interferon }
\end{array}\right.
$$

O modelo utilizado, a exemplo da seção anterior, é:

$$
\alpha_{i j}\left(t \mid x_{i j}\right)=\alpha_{0}(t) e^{\eta_{i j}}
$$

com $\eta_{i j}=x_{i j} \beta_{j}=x_{i j}\left(\beta+u_{j}\right)$ e o índice $i j$ representando a $i$-ésima infecção (unidade do nível 1) do $j$-ésimo paciente (unidade do nível 2). Supõe-se que as variáveis $u_{j}$ ( $j=$ $1, \ldots, 128)$ são independentes e apresentam distribuição normal com média zero e variância $\sigma^{2}$. A Tabela 4.4 apresenta os resultados obtidos.

Observando os resultados obtidos para o parâmetro da variância, pode-se verificar que tanto no caso da MQL quanto da PQL existem fortes indícios de que o modelo supondo independência não é adequado. Os p-valores obtidos, tanto pelo bootstrap quanto supondo a distribuição de Qui-quadrado para a estatística de Wald, são pequenos e levam à mesma conclusão. O intervalo de $95 \%$ de confiança para $\sigma^{2}$ obtido pelo bootstrap no caso da PQL foi $(0,1172 ; 0,6435)$, que não contém o valor zero. Neste caso, utilizou-se 800 replicações bootstrap. O número de replicações foi menor do que no caso anterior para diminuir um pouco o tempo de execução do programa. 


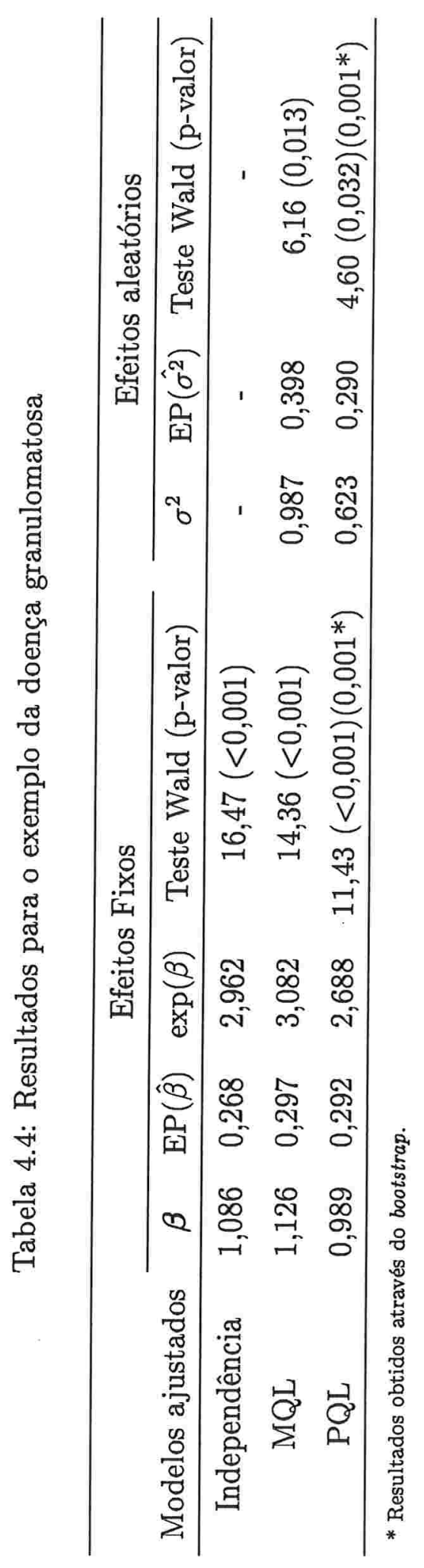


Com relação ao parâmetro fixo, pode-se verificar que o maior erro padrão da MQL é maior do que aquele obtido pela $\mathrm{PQL}$, sugerindo que o procedimento $\mathrm{PQL}$ é um pouco mais preciso. Os níveis descritivos dos testes são todos muito pequenos, indicando a existência de diferença dos tratamentos.

As correções da $\mathrm{PQL}$ não estão apresentadas na Tabela 4.4 porque houve um problema computacional. A forma proposta para a obtenção das estimativas da quase verossimilhança penalizada com a correção do viés resulta em cálculos envolvendo matrizes de dimensões muito grandes. Neste conjunto de dados, são 128 pacientes, mas no total temos 203 observações. Quando criamos as variáveis de Poisson para o caso do modelo de Cox, ficamos com mais de 9 mil observações. Isso acontece devido ao número pequeno de empates e censuras. Para dados com grande número de observações, em um certo momento da execução do programa do cálculo das correções, os cálculos são interrompidos devido a um erro, que trava o computador. Por esse motivo as estimativas corrigidas não foram obtidas.

Neste caso também foi feito o ajuste dos modelos exponencial e Weibull. Considerouse, em ambos os casos, apenas o intercepto aleatório, ou seja, o preditor linear é dado por $\eta_{i j}=\alpha_{j}+\beta x_{i j}=\alpha+b_{j}+\beta x_{i j}$, em que $b_{j}, j=1, \ldots, 128$, são variáveis aleatórias independentes com distribuição normal de média zero e variância $\sigma^{2}$. A covariável $x_{i j}$ representa o tratamento ( 1 se placebo e 0 se gama interferon) $i$-ésima infeç̧ão do $j$-ésimo paciente. Os resultados estão apresentados nas tabelas 4.5 e 4.6.

Neste exemplo, o método MQL também apresentou problemas e não convergiu em 50 iterações. 
Tabela 4.5: Resultados do ajuste exponencial para o exemplo da doença granulomatosa

\begin{tabular}{|c|c|c|c|}
\hline \multirow[b]{2}{*}{ Parâmetros } & \multicolumn{3}{|c|}{ Modelos } \\
\hline & Independência & Multinível - MQL & Multinível - PQL \\
\hline$\alpha$ & $-6,8529$ & $-6,8619$ & $-6,9190$ \\
\hline $\operatorname{EP}(\hat{\alpha})$ & 0,2236 & 0,2565 & 0,2476 \\
\hline Teste Wald (p-valor) & $939,42(<0,001)$ & $715,56(<0,001)$ & $781,20(<0,001)$ \\
\hline$\beta$ & 1,0544 & 1,0343 & 0,9814 \\
\hline $\mathrm{EP}(\hat{\beta})$ & 0,3149 & 0,3416 & 0,3014 \\
\hline Teste Wald (p-valor) & $16,4025(<0,001)$ & $10,7584(0,001)$ & $10,6276(0,001)$ \\
\hline$\sigma^{2}$ & - & 0,9400 & 0,5963 \\
\hline $\operatorname{EP}\left(\hat{\sigma^{2}}\right)$ & - & 0,3246 & 0,2302 \\
\hline Teste Wald (p-valor) & - & $8,41(0,004)$ & $6,7081(0,009)$ \\
\hline
\end{tabular}

Tabela 4.6: Resultados do ajuste do modelo Weibull para o exemplo da doença granulomatosa

\begin{tabular}{|c|c|c|}
\hline \multirow[b]{2}{*}{ Parâmetros } & \multicolumn{2}{|c|}{ Modelos } \\
\hline & Independência & Multinível - PQL \\
\hline$\alpha$ & $-6,2284$ & $-7,0170$ \\
\hline $\operatorname{EP}(\hat{\alpha})$ & 0,2236 & 0,2482 \\
\hline Teste Wald (p-valor) & $1221,50(<0,001)$ & $799,1929(<0,001)$ \\
\hline$\beta$ & 1,0280 & 0,9848 \\
\hline $\operatorname{EP}(\hat{\beta})$ & 0,2605 & 0,3024 \\
\hline Teste Wald (p-valor) & $15,6025(<0,001)$ & $10,6276(0,001)$ \\
\hline$\sigma^{2}$ & - & 0,6110 \\
\hline $\operatorname{EP}\left(\hat{\sigma^{2}}\right)$ & - & 0,2327 \\
\hline Teste Wald (p-valor) & - & $6,9169(0,008)$ \\
\hline
\end{tabular}

A estimativa de $\lambda$ (expr. (3.6)) obtida no modelo de independência é 0,88775 e no modelo multinível é 0,88773 . 


\subsection{Avaliação de uma droga carcinogênica em ratos}

Este estudo foi um experimento com ratos, para verificar o efeito de uma droga carcinogênica, publicados por Mantel, Bohidar \& Ciminera (1977). No total, foram utilizadas 50 ninhadas de ratos. Selecionaram-se, de cada ninhada, três ratas: 2 delas receberam a droga e a terceira recebeu placebo. A variável reposta observada é o tempo até o aparecimento do tumor.

Note que é conveniente supor que há alguma dependência entre ratas da mesma ninhada. Assim sendo, cada rata é considerada uma unidade do nível 1 e as ninhadas são as unidades do nível 2. A covariável utilizada é tratamento, que foi definida da seguinte maneira:

$$
\text { Tratamento }=\left\{\begin{array}{l}
1 \text { Droga } \\
0 \text { Placebo }
\end{array}\right.
$$

O modelo ajustado é o mesmo utilizado nos dois casos anteriores. A Tabela 4.7 apresenta os resultados.

Neste exemplo, ocorreu um problema na estimação do parâmetro da variância, tanto por meio da quase-verossimilhança marginal, quanto pela quase verossimilhança penalizada. Em todos os casos, obteve-se o valor zero e o erro padrão não foi calculado. Esse problema ocorre na macro GLIMMIX e não foi possível identificar a causa. Se a variância é zero, então é claro que as estimativas serão todas iguais às obtidas no modelo de independência. Além disso, não é possível realizar os testes de hipóteses para o parâmetro da variância.

$\mathrm{Na}$ análise feita utilizando o modelo de fragilidade gama, os testes indicam que o modelo de independência é adequado, ou seja, não há dependência estatisticamente significante entre observações de uma mesma ninhada. Dessa forma, a conclusão obtida pela modelagem multinível é coerente, mas ainda assim esse problema da estimativa zero 


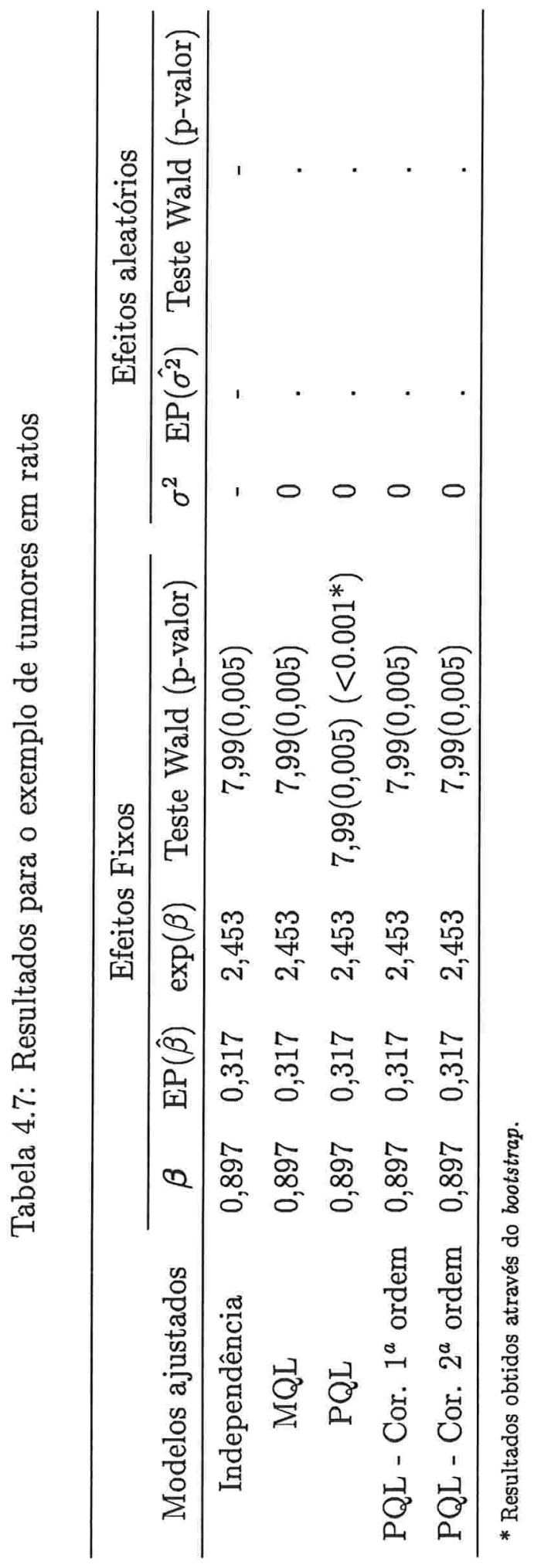


do parâmetro da variância deve ser investigado.

Outro problema que ocorreu neste exemplo foi com relação ao bootstrap no caso da MQL. Em muitas amostras não houve convergência do algoritmo da MQL. No caso da $\mathrm{PQL}$, foram poucos os casos e foi possível fazer os cálculos. Assim, obteve-se o intervalo de $95 \%$ de confiança para $\beta$ apenas no caso da PQL, baseado em 1000 replicações bootstrap: $(0,5064 ; 1,3133)$. Esse resultado indica que há efeito de tratamento estatisticamente significante.

Finalmente, foi feito o ajuste dos dados utilizando-se os modelos paramétricos. O coeficiente aleatório considerado foi também o intercepto. Assim, da mesma forma como nos exemplos anteriores, temos $\eta_{i j}=\alpha_{j}+\beta x_{i j}=\alpha+b_{j}+\beta x_{i j}$, em que $b_{j}$ são variáveis aleatórias normalmente distribuídas e a covariável $x_{i j}$ representa o tratamento ( 1 se droga e 0 placebo) da $i$-ésima rata da $j$-ésima ninhada. Os resultados estão apresentados nas tabelas 4.8 e 4.9 .

Pode-se notar que este foi o único exemplo em que o modelo multinível com distribuição Weibull convergiu quando a abordagem MQL foi utilizada. Todos os testes feitos para o coeficiente aleatório indicam que o modelo de independência é adequado. 
Tabela 4.8: Resultados do ajuste exponencial para o exemplo de tumores em ratos

\begin{tabular}{|c|c|c|c|}
\hline \multirow[b]{2}{*}{ Parâmetros } & \multicolumn{3}{|c|}{ Modelos } \\
\hline & Independência & Multinível - MQL & Multinível - PQL \\
\hline$\alpha$ & $-6,167$ & $-6,166$ & $-6,916$ \\
\hline $\operatorname{EP}(\hat{\alpha})$ & 0,229 & 0,230 & 0,231 \\
\hline Teste Wald (p-valor) & $722,53(<0,001)$ & $716,6329(<0,001)$ & $715,03(<0,001)$ \\
\hline$\beta$ & 0,832 & 0,832 & 0,832 \\
\hline $\operatorname{EP}(\hat{\beta})$ & 0,317 & 0,316 & 0,317 \\
\hline Teste Wald (p-valor) & $6,92(0,008)$ & $6,92(0,008)$ & $6,92(0,008)$ \\
\hline$\sigma^{2}$ & - & 0,025 & 0,027 \\
\hline $\operatorname{EP}\left(\hat{\sigma^{2}}\right)$ & - & 0,274 & 0,270 \\
\hline Teste Wald (p-valor) & - & $0,01(0,463)$ & $0,01(0,460)$ \\
\hline
\end{tabular}

Tabela 4.9: Resultados do ajuste do modelo Weibull para o exemplo de tumores em ratos

\begin{tabular}{|c|c|c|c|}
\hline \multirow[b]{2}{*}{ Parâmetros } & \multicolumn{3}{|c|}{ Modelos } \\
\hline & Independência & Multinível - MQL & Multinível - PQL \\
\hline$\alpha$ & $-18,875$ & $-23,761$ & $-19,443$ \\
\hline $\operatorname{EP}(\hat{\alpha})$ & 0,229 & 0,242 & 0,249 \\
\hline Teste Wald (p-valor) & $6768,35(<0,001)$ & $9664,86(<0,001)$ & $6093,36(<0,001)$ \\
\hline$\beta$ & 0,905 & 0,932 & 0,908 \\
\hline $\operatorname{EP}(\hat{\beta})$ & 0,317 & 0,308 & 0,322 \\
\hline Teste Wald (p-valor) & $8,18(0,004)$ & $9,18(0,002)$ & $7,95(0,004)$ \\
\hline$\sigma^{2}$ & - & 0,457 & 0,428 \\
\hline $\operatorname{EP}\left(\hat{\sigma^{2}}\right)$ & - & 0,499 & 0,386 \\
\hline Teste Wald (p-valor) & - & $0,83(0,363)$ & $1,23(0,267)$ \\
\hline
\end{tabular}

A estim. de $\lambda$ (exp. (3.6)) obtida no modelo de indep. é 3,78, no multinível-MQL é 4,87 e no multinível-PQL é 3,91. 


\section{Capítulo 5}

\section{Conclusão}

Nesta dissertação, o problema da dependência entre observações em dados de sobrevivência foi abordado através dos modelos multiníveis. Para a estimação dos parâmetros, utilizou-se o resultado que mostra a equivalência da verossimilhança dos modelos de sobrevivência com a verossimilhança de Poisson de variáveis artificialmente criadas. Com isso, foram apresentados basicamente dois métodos de estimação de modelos multiníveis lineares generalizados - PQL e MQL.

Um problema da utilização da equivalência das verossimilhanças de Poisson e dos modelos de sobrevivência ocorre quando está se trabalhando com o modelo de Cox. Quando se criam as variáveis de Poisson para o modelo de Cox, o conjunto de dados que é de fato utilizado para a estimação via PQL ou MQL fica com uma quantidade muito grande de observações. Por exemplo, no caso do exemplo da doença granulomatosa crônica da infância, os dados iniciais têm 203 observações e, quando se criam as variáveis auxiliares, o conjunto de dados utilizado na estimação tem pouco mais de 9000 observações. O número de observações criadas depende do número de tempos de falhas distintos. Por esse motivo, tornou-se inviável o cálculo das estimativas corrigidas da PQL para este caso. 
Com base nos exemplos analisados, foi possível perceber que o método MQL apresenta mais problemas de convergência do que o PQL. Com relação às estimativas corrigidas da quase verossimilhança penalizada, os ganhos não foram muito significativos para os exemplos tratados que justifique o trabalho adicional para o cálculo das correções. Portanto, o método PQL se mostrou a escolha mais natural para estimar os parâmetros dos modelos. Seria muito interessante em um trabalho futuro comparar também os métodos descritos neste trabalho com o método proposto por Vaida \& Xu (2000). Os autores trabalham com modelos de riscos proporcionais e utilizam o algoritmo EM para a estimação dos parâmetros. Além disso, como os exemplos trabalhados possuem apenas variáveis binárias, seria interessante verificar como se comportam esses métodos de estimação quando se tem covariáveis contínuas no modelo e, ainda, mais de um efeito aleatório.

Uma questão importante é com relação à distribuição dos efeitos aleatórios. Neste trabalho, utilizou-se a distribuição normal, mas ela pode não ser adequada. Seria interessante generalizar os resultados para que outras distribuições fossem possíveis e ter alguma forma de analisar a adequação do modelo para verificar qual distribuição dos efeitos aleatórios é mais adequada.

Além da questão da distribuição dos efeitos aleatórios, seria interessante também desenvolver métodos de diagnósticos para verificar se a suposição de riscos proporcionais está sendo satisfeita. Alguns dos problemas observados neste trabalho podem ter ocorrido devido à inadequação do modelo utilizado. Ainda, se a suposição de proporcionalidade dos riscos for satisfeita, seria interessante métodos para verificar qual modelo é mais adequado (paramétrico ou semiparamétrico).

Por fim, com relação aos testes de hipóteses, há muito o que ser desenvolvido. O bootstrap é uma alternativa interessante, mas existe o problema computacional. No caso da regressão de Cox, trabalha-se com conjuntos de dados grandes, conforme já mencionado, e o tempo computacional é grande. No caso Weibull, o programa para a estimação 
é um pouco mais complicado, pois tem um parâmetro extra a ser estimado, e não foi possível utilizar o bootstrap por problemas computacionais. Conforme já dito, está sendo desenvolvido um teste do escore para os parâmetros da variância dos efeitos aleatórios para o caso da distribuição de Weibull. 


\section{Apêndice A}

\section{Cálculo da expressão aproximada da PQL}

Considere a expressão (3.14) escrita na forma $c|\mathbf{D}|^{-1 / 2} \int e^{-\mathbf{k}(\mathbf{b})} d \mathbf{b}$ e sejam $\mathbf{k}^{\prime}$ e $\mathbf{k}^{\prime \prime}$ o vetor e a matriz $q \times q$ de derivadas primeiras e segundas de $\mathbf{k}(\mathbf{b})$ com relação a $\mathbf{b}$, respectivamente. Ignorando a constante $c$ e utilizando a aproximação de Laplace, tem-se:

$$
q l(\boldsymbol{\beta}, \boldsymbol{\theta}) \approx-\frac{1}{2} \log |\mathbf{D}|-\frac{1}{2} \log \left|\mathbf{k}^{\prime \prime}(\tilde{\mathbf{b}})\right|-\mathbf{k}(\tilde{\mathbf{b}}),
$$

em que $\tilde{\mathbf{b}}=\tilde{\mathbf{b}}(\boldsymbol{\beta}, \boldsymbol{\theta})$ representa a solução de $\mathbf{k}^{\prime}(\mathbf{b})=0$.

Derivando-se $\mathbf{k}$ com relação a $\mathbf{b}$, tem-se:

$$
\mathbf{k}^{\prime}(\mathbf{b})=\sum_{i=1}^{n} \frac{\partial d_{i}}{\partial \mathbf{b}}+\frac{1}{2} 2 \mathbf{D}^{-1} \mathbf{b}
$$

Calculando $\frac{\partial d_{i}}{\partial \mathbf{b}}$, tem-se: 


$$
\begin{aligned}
\frac{\partial d_{i}}{\partial \mathbf{b}} & =\frac{\left(y_{i}-\mu_{i}^{b}\right)}{a_{i} v\left(\mu_{i}^{b}\right)} \frac{\partial \mu_{i}^{b}}{\partial \mathbf{b}}=\frac{\left(y_{i}-\mu_{i}^{b}\right)}{a_{i} v\left(\mu_{i}^{b}\right)} \frac{\partial \mu_{i}^{b}}{\partial \eta_{i}} \frac{\partial \eta_{i}}{\partial \mathbf{b}} \\
& =\frac{y_{i}-\mu_{i}^{b}}{a_{i} v\left(\mu_{i}^{b}\right)}\left(g_{-1}\left(\eta_{i}\right)\right)^{\prime} \mathbf{z}_{i}=\frac{\left(y_{i}-\mu_{i}^{b}\right)}{a_{i} v\left(\mu_{i}^{b}\right)} \frac{1}{g^{\prime}\left(\mu_{i}^{b}\right)} \mathbf{z}_{i}
\end{aligned}
$$

Assim, a derivada de $\mathbf{k}(\mathbf{b})$ em relação a b é dada por:

$$
\begin{aligned}
\mathbf{k}^{\prime}(\mathbf{b}) & =\sum_{i=1}^{n} \frac{\partial d_{i}}{\partial \mathbf{b}}+\mathbf{D}_{-\mathbf{1}} \mathbf{b}= \\
& =-\sum_{i=1}^{n} \frac{\left(y_{i}-\mu_{i}^{b}\right) \mathbf{z}_{i}}{\phi a_{i} v\left(\mu _ { i } ^ { b } g ^ { \prime } \left(\mu_{i}^{b}\right.\right.}+\mathbf{D}^{-1} \mathbf{b}
\end{aligned}
$$

Portanto, $\tilde{\mathbf{b}}$ é tal que:

$$
\mathbf{k}^{\prime}(\mathbf{b})=-\sum_{i=1}^{n} \frac{\left(y_{i}-\mu_{i}^{b}\right) \mathbf{z}_{i}}{\phi a_{i} v\left(\mu_{i}^{b}\right) g^{\prime}\left(\mu_{i}^{b}\right)}+\mathbf{D}^{-1} \mathbf{b}=0
$$

A derivada segunda de $\mathbf{k}$ é dada por:

$$
\mathbf{k}^{\prime \prime}(\mathbf{b})=\mathbf{D}^{-1}-\sum_{i=1}^{n}\left[\frac{\mathbf{z}_{i}}{\phi a_{i} v\left(\mu_{i}^{b}\right) g^{\prime}\left(\mu_{i}^{b}\right)} \frac{\mathbf{z}_{i}^{t}}{g^{\prime}\left(\mu_{i}^{b}\right)}+\left(y_{i}-\mu_{i}^{b}\right) \mathbf{z}_{i} \frac{\partial}{\partial \mathbf{b}}\left(\frac{1}{\phi a_{i} v\left(\mu_{i}^{b}\right) g^{\prime}\left(\mu_{i}^{b}\right)}\right)\right] \text {. }
$$

Pode-se então escrever:

em que

$$
\mathbf{k}^{\prime \prime}(\mathbf{b})=\sum_{i=1}^{n} \frac{\mathbf{z}_{i} \mathbf{z}_{i}^{t}}{\phi a_{i} v\left(\mu_{i}^{b}\right)\left(g^{\prime}\left(\mu_{i}^{b}\right)\right)^{2}}+\mathbf{D}^{-1}+R,
$$

$$
R=-\sum_{i=1}^{n}\left(y_{i}-\mu_{i}^{b}\right) \mathbf{z}_{i} \frac{\partial}{\partial \mathbf{b}}\left(\frac{1}{\phi a_{i} v\left(\mu_{i}^{b}\right) g^{\prime}\left(\mu_{i}^{b}\right)}\right)
$$




$$
\begin{aligned}
& \text { Fazendo } W=\operatorname{diag}\left[\left(\phi a_{i} v\left(\mu_{i}^{b}\right)\left(g^{\prime}\left(\mu_{i}^{b}\right)\right)^{2}\right)^{-1}\right] \text {, então } \\
& \qquad \begin{array}{l}
\mathbf{k}^{\prime \prime}(\mathbf{b})=Z^{t} W Z+\mathbf{D}^{-1}+R .
\end{array}
\end{aligned}
$$

Note que, quando a ligação é canônica, então tem-se $R=0$, pois $v\left(\mu_{i}^{b}\right)=\frac{\partial \mu_{i}^{b}}{\partial \eta_{i}^{b}} \mathrm{e}$ $g^{\prime}\left(\mu_{i}^{b}\right)=\frac{\partial \eta_{i}^{b}}{\partial \mu_{i}^{b}}$. Ainda, para qualquer função de ligação, $R$ tem esperança igual a zero. Assim, ignorando o termo $R$, tem-se $\mathbf{k}^{\prime \prime}(\mathbf{b})=Z^{t} W Z+\mathbf{D}^{-1}$. Substituindo estes resultados em (3.16), então chega-se à expressão (3.17). 


\section{Referências Bibliográficas}

Aitkin, M., Anderson, D., Francis, B. \& Hinde, J. (1989). Statistical Modelling in GLIM, Clarendon Press, Oxford.

Aitkin, M., Anderson, D. \& Hinde, J. (1981). Statistical modelling of data on teaching styles, Journal of the Royal Statistical Society, Ser. A 144(4): 419-461.

Barndorff-Nielsen, O. E. \& Cox, D. R. (1989). Asymptotic Techniques for use in Statistics, Chapman and Hall, London.

Bennet, N. (1976). Teaching Styles and Pupil progress, Open Books, London.

Breslow, N. E. (1974). Covariance analysis of censored survival data, Biometrics 30: 8999.

Breslow, N. E. \& Clayton, D. G. (1993). Approximate inference in generalized linear mixed models, Journal of the American Statistical Association 88: 9-25.

Breslow, N. E. \& Lin, X. (1995). Bias correction in generalised linear mixed models with a single component of dispersion, Biometrika 82(1): 81-91.

Chicarino, M. P. Z. (1999). Modelo semiparamétrico de fragilidade gama, Dissertação de mestrado, Universidade de São Paulo, Intituto de Matemática e Estatística. 
Clayton, D. (1978). A model for association in bivariate life tables and its application in epidemiologocal studies of familial tendency in chronic disease incidence, Biometrika 65: $141-151$.

Cox, D. R. (1972). Regression-models and life-tables, Journal of the Royal Statistical Society, Ser. B 34: 187-220.

Cox, D. R. \& Oakes, D. (1984). Analysis of survival data, Chapman and Hall, London.

Dempster, A. P., Laird, N. M. \& Rubin, D. B. (1977). Maximum likelihood from incomplete data via the em algorithm, Journal of the Royal Statistical Society, Ser. B 39: $1-38$.

Draper, D. (1995). Inference and hierarquical modeling in the social sciences, Journal of Educational and Behavioral Statistics 20(2): 115-147.

Efron, B. \& Tibshirani, R. J. (1993). An introduction to the bootstrap, Chapman and Hall.

Fleming, T. R. \& Harrington, D. P. (1991). Counting Processes and Survival Analysis, John Wiley and Sons.

Gilmour, A. R., Anderson, R. D. \& Rae, A. L. (1985). The analysis of binomial data by a generalized linear mixed model, Biometrika 72(3): 593-599.

Goldstein, H. (1986). Multilevel mixed linear model analysis using iterative generalized least squares, Biometrika 73: 43-56.

Goldstein, H. (1991). Nonlinear multilevel model, with an application to discrete response data, Biometrika 78: 45-51.

Goldstein, H. (1995). Multilevel Statistical Models, $2^{\mathrm{a}}$ edn, Kendall's Library of Statistics, London. 
Grande, R. H. M., Lima, A. C. P., Filho, L. E. R. \& Witzel, M. F. (2000). Clinical evaluation of an adhesive used as a fissure sealant, American Journal of Dentistry 13(4): 167-170.

Graybill, F. A. (1969). Matrices with applications in Statistics, $2^{\mathrm{a}}$ edn, Wadsworth $\mathrm{Pu}-$ blishing Company, Inc, Belmont.

Harville, D. A. (1977). Maximum likelihood aproaches to variance component estimation and to related problems, Journal of the American Statistical Association 73: 320-327.

Hougaard, P. (2000). Analysis of Multivariate Survival Data, Springer-Verlag, New York.

Hox, J. J. (1995). Applied multilevel analysis, TT-Publikaties, Amsterdam.

Klein, J. P. \& Moeschberger, M. L. (1997). Survival analysis - Techniques for censored and truncated data, Springer-Verlag, New York.

Lee, Y. \& Nelder, J. A. (1996). Hierarquical generalized linear models, Journal of the Royal Statistical Society, Ser. B 58(4): 619-678.

Lin, X. (1997). Variance component testing in generalised linear models with random effects, Biometrika 84(2): 309-326.

Lin, X. \& Breslow, N. E. (1996). Bias correction in generalised linear mixed models with multiple components of dispersion, Journal of the American Statistical Association 91: $1007-1016$.

Lindley, D. \& Smith, A. F. M. (1972). Bayes estimates of the linear model (with discussion), Journal of the Royal Statistical Society, Ser. B 34: 1-41.

Longford, N. T. (1987). A fast scoring algorithm for maximum likelihood estimation in unbalanced mixed models with nested random effects, Biometrika 74: 817-827. 
Mantel, N., Bohidar, N. R. \& Ciminera, J. L. (1977). Mantel-Haenzel analysis of litter matched time-to-response data, with modifications for recovery of interlitter information, Cancer research 37: 3863-3868.

McCullagh, P. \& Nelder, J. (1989). Generalized Linear Models, $2^{\mathrm{a}}$ edn, Chapman and Hall, London.

Schall, R. (1991). Estimation in generalized linear models with random effects, Biometrika 78(4): 719-727.

Solomon, P. J. \& Cox, D. R. (1992). Nonlinear component of variance models, Biometrika 79(1): 1-11.

Vaida, F. \& Xu, R. (2000). Proportional hazards model with random effects, Statistics in Medicine 19: 3309-3324.

Walker, S. (1996). An EM algorithm for nonlinear random effects models, Biometrics 52: 934-944.

Zeger, L. S. \& Karim, M. R. (1991). Generalized linear models with random effects: a Gibbs sampling approach, Journal of the American Statistical Association 86: 79-86.

Zitelli, B. J. \& Holly, W. D. (1992). Diagnóstico clínico em Pediatria, 2a edn, Editora Manole Ltda, São Paulo. 